\title{
PD-L1- and calcitriol-dependent liposomal antigen-specific regulation of systemic inflammatory autoimmune disease
}

Ryan Galea, ${ }^{1}$ Hendrik J. Nel, ${ }^{1}$ Meghna Talekar, ${ }^{1}$ Xiao Liu, ${ }^{1}$ Joshua D. Ooi, ${ }^{2}$ Megan Huynh, ${ }^{2}$ Sara Hadjigol, ${ }^{2}$ Kate J. Robson, ${ }^{2}$ Yi Tian Ting, ${ }^{3}$ Suzanne Cole, ${ }^{4}$ Karyn Cochlin, ${ }^{4}$ Shannon Hitchcock, ${ }^{4}$ Bijun Zeng, ${ }^{1}$ Suman Yekollu, ${ }^{1}$ Martine Boks, ${ }^{1}$ Natalie Goh, ${ }^{1}$ Helen Roberts, ${ }^{5}$ Jamie Rossjohn,,${ }^{3,6,7}$ Hugh H. Reid, ${ }^{3}$ Ben J. Boyd, ${ }^{8}$ Ravi Malaviya, ${ }^{4}$ David J. Shealy, ${ }^{4}$ Daniel G. Baker, ${ }^{4}$ Loui Madakamutil, ${ }^{4}$ A. Richard Kitching, ${ }^{2,9}$ Brendan J. O'Sullivan, ${ }^{1}$ and Ranjeny Thomas'

'The University of Queensland Diamantina Institute, The University of Queensland, Woolloongabba, Queensland, Australia. ${ }^{2}$ Centre for Inflammatory Diseases, Monash University Department of Medicine, Monash Medical Centre, Clayton, Victoria, Australia. Infection and Immunity Program and Department of Biochemistry and Molecular Biology, Biomedicine Discovery Institute, Monash University, Clayton, Victoria, Australia. ${ }^{4}$ Discovery Immunology, Janssen Research \& Development LLC, Spring House, Pennsylvania, USA. ${ }^{5}$ Dendright, Brisbane, Queensland, Australia. ${ }^{6}$ Australian Research Council Centre of Excellence in Advanced Molecular Imaging, Monash University, Clayton, Victoria, Australia. ${ }^{7}$ Institute of Infection and Immunity, School of Medicine, Cardiff University, Cardiff, United Kingdom. ${ }^{8}$ Drug Delivery, Disposition and Dynamics and Australian Research Council Centre of Excellence in Convergent Bio-Nano Science and Technology, Monash Institute of Pharmaceutical Sciences, Parkville, Victoria, Australia. ${ }^{9}$ Departments of Nephrology and Paediatric Nephrology, Monash Health, Clayton, Victoria, Australia.

Authorship note: RG, HJN, MT, and XL contributed equally to this work.

Conflict of interest: RT has filed provisional patents surrounding technology for targeting DCs for antigen-specific tolerance (US patent 9,017,697 B2: 2006, PCT/ AU2013/000303) and is a director of the spin-off company, Dendright which developed immunotherapy to target DCs to suppress rheumatoid arthritis in collaboration with Janssen Biotech Inc. RG, HJN, SY, BZ, BJO, and HR were supported in part from by Janssen Biotech Inc., and SC, KC, SH, $R M, D J S, D C B$, and $L M$ were employees of Janssen Research \& Development.

Copyright: (c) 2019, American Society for Clinical Investigation.

Submitted: November 6, 2018

Accepted: August 21, 2019

Published: September १९, 2019.

Reference information: /CI Insight.

2019;4(18):e126025.

https://doi.org/10.1172/jci.

insight.126025.
Autoimmune diseases resulting from MHC class II-restricted autoantigen-specific $\mathrm{T}$ cell immunity include the systemic inflammatory autoimmune conditions rheumatoid arthritis and vasculitis. While currently treated with broad-acting immunosuppressive drugs, a preferable strategy is to regulate antigen-specific effector T cells (Teffs) to restore tolerance by exploiting DC antigen presentation. We targeted draining lymph node (dLN) phagocytic DCs using liposomes encapsulating 1 $\alpha, 25$-dihydroxyvitamin D3 (calcitriol) and antigenic peptide to elucidate mechanisms of tolerance used by DCs and responding T cells under resting and immunized conditions. PD-L1 expression was upregulated in dLNs of immunized relative to naive mice. Subcutaneous administration of liposomes encapsulating $\mathrm{OVA}_{323-339}$ and calcitriol targeted dLN PD-L1 ${ }^{\text {hi }}$ DCs of immunized mice and reduced their MHC class II expression. OVA ${ }_{323-339} /$ calcitriol liposomes suppressed expansion, differentiation, and function of Teffs and induced Foxp ${ }^{+}$and IL-10+ peripheral Tregs in an antigen-specific manner, which was dependent on PD-L1. Peptide/ calcitriol liposomes modulated CD40 expression by human DCs and promoted Treg induction in vitro. Liposomes encapsulating calcitriol and disease-associated peptides suppressed the severity of rheumatoid arthritis and Goodpasture's vasculitis models with suppression of antigen-specific memory T cell differentiation and function. Accordingly, peptide/calcitriol liposomes leverage DC PD-L1 for antigen-specific $T$ cell regulation and induce antigen-specific tolerance in inflammatory autoimmune diseases.

\section{Introduction}

Systemic inflammatory autoimmune conditions, such as rheumatoid arthritis (RA) and the small-vessel vasculitides, are characterized by persistent widespread tissue inflammation, shortened life span, and end-organ damage, such as joint destruction and kidney failure. Current treatments include inhibitors of TNF and IL-6, CTLA4-Ig, anti-CD20, glucocorticoids, and cytotoxic drugs, particularly those targeting the tissue-damaging disease effector phase $(1,2)$. While limiting symptoms, these therapies are associated with adverse effects, are not curative, and fail to fully control disease in most patients (3). Autoantigen-specific immunological tolerance is a preferable strategy to control both cellular and humoral immune responses in autoimmune disease. 
DCs, as essential regulators of both innate and adaptive immunity (4), represent a powerful natural immune target for induction of antigen-specific tolerance (5). The NF- $\mathrm{kB}$ subunit RelB regulates DC function (6): in RelB-deficient splenic DCs or human tolerogenic monocyte-derived DCs costimulatory molecule upregulation is refractory to TLR-ligand activation $(7,8)$. Adoptively transferred antigen-exposed RelB- or CD40-deficient DCs suppressed murine immune responses, including autoimmune arthritis, in an antigen-specific manner (9-11). Furthermore, in a phase I clinical trial in HLA-DR high-risk genotype-positive RA patients, intradermally injected autologous DCs exposed to citrullinated autoantigenic peptides and the NF- $\mathrm{KB}$ inhibitor BAY11-7082 were safe, reduced circulating CD4 ${ }^{+}$effector T cells (Teffs), and increased the ratio of Tregs to Teffs (12). While this trial provided an important clinical proof of concept, simpler strategies targeting DCs in situ are needed for widespread clinical use. We and others have shown that PLGA, gold, or liposome nanoparticles encapsulating antigen and immunomodulators, such as rapamycin, curcumin, or aryl hydrocarbon receptor ligand, induce antigen-specific tolerance in animal models of immunity and autoimmune disease (13-16). However, for clinical translation, particle format, inhibitor, and antigen are important considerations. Furthermore, while disease-associated autoimmune responses, including autoreactive $\mathrm{T}$ cells and autoantibodies, are often detectable years before onset of autoimmune rheumatic diseases $(17,18)$, it is currently unclear how tolerizing immunotherapeutic interventions would regulate systemic inflammatory autoimmune diseases driven by autoantibody-mediated innate immune mechanisms and autoreactive $\mathrm{T}$ cells specific for self-antigens with widespread tissue expression. However, models of transgenic self-antigen demonstrated that, when self-antigen was widely expressed, CD4 T cell tolerance was maintained by thymic deletion of high-affinity self-reactive $\mathrm{T}$ cells and persistent unresponsiveness of residual low-affinity $\mathrm{T}$ cells with low-intensity staining of peptide-MHC tetramer in the periphery $(19,20)$.

Skin draining lymph nodes (dLNs) contain CD11 $\mathrm{c}^{+} \mathrm{CD} 11 \mathrm{~b}^{+}$Ly6C $\mathrm{C}^{-}$dermal migratory $\mathrm{DCs}, \mathrm{CD} 40^{\text {hi }}$ Langerhans cells migrating from the dermis and epidermis through afferent lymphatics to the subcapsular sinus, and $\mathrm{CD} 11 \mathrm{c}^{+} \mathrm{CD} 8{ }^{+} \mathrm{DEC} 205^{+} \mathrm{CD} 11 \mathrm{~b}^{-}$resident DCs derived from the blood. Genetic or antibody approaches targeting antigen to specific LN DC populations demonstrated that CD $11 b^{+}$LN DCs mediate antigen-specific peripheral tolerance $(21,22)$. In inflammatory settings, migratory DCs increase in dLNs and transport s.c. injected material to the T cell area $(23,24)$. Antigen-presenting DCs are also recruited from perinodal adipose tissue to inflamed LN (25). LN draining inflamed joints have greater lymphangiogenesis and lymph flow and contain larger numbers of inflammatory monocyte-derived DCs (moDCs) and CD11 b myeloid cells $(26,27)$. Furthermore, tolerance to dermal vaccination is impaired in mice lacking dermal lymphatic drainage (28).

Liposomes have an established record in the clinic for targeted delivery of lipophilic drugs (29) and provide a versatile platform for codelivery to immune cells of hydrophilic or hydrophobic payloads (16, 30, 31 ). While liposomes coencapsulating protein antigen and curcumin or BAY11-7082 suppressed NF- $\mathrm{kB}$ in dLNs and regulated immunity in a CD25-dependent manner in proof-of-concept studies, curcumin is classified as a pan-assay interference compound candidate and is unsuitable for clinical translation due to its instability and reactivity (32). On the other hand, 1 $\alpha, 25$-dihydroxyvitamin D3 (calcitriol) has an established clinical track record and is of particular interest in DC biology, as RelB is selectively suppressed downstream of the vitamin D receptor (VDR) (33) through a mechanism involving direct binding of VDR/ $\mathrm{RXR} \alpha$ to a defined region of the RelB promoter (34). Therefore, we developed L- $\alpha$-egg phosphatidylcholine (EPC) and L- $\alpha$-egg phosphatidylglycerol (EPG) liposomes encapsulating peptide antigen and calcitriol. We studied pharmacology and mechanisms of action through passive targeting of dLN phagocytic DCs to define the mechanisms of tolerance used by targeted DCs and responding T cells in the naive or antigen-experienced state and in experimental inflammatory autoimmune diseases.

\section{Results}

Passive liposomal targeting of myeloid DCs in resting or inflamed skin dLNs after s.c. injection. To assess uptake, distribution, and function, we generated liposomes encapsulating calcitriol and the model antigen, $\mathrm{OVA}_{323-339}$ peptide. Liposomes were 105-135 $\mathrm{nm}$ diameter with a surface charge of (-) $55 \mathrm{mV}$ and were stable in HEPES buffer $\mathrm{pH}$ 7.4 for at least 3 months at -30 and $4^{\circ} \mathrm{C}$ (Figure 1A). We incorporated Texas red-labeled (TXR-labeled) lipid and BODIPY-fluorescein-labeled calcitriol into $\mathrm{OVA}_{323-339} /$ calcitriol liposomes (Figure 1B). After incubation of the RAW 264.7 macrophage cell line with dual-labeled liposomes for 2 hours, both labels were observed intracellularly (Figure 1C). OVA $323-339$ /calcitriol liposomes labeled with DiI (100 $\mu$ l) were injected s.c. at the tail base into naive BALB/c mice or mice primed with OVA/QuilA at the tail base 3 days previously. One day after s.c. injection, fluorescent signal was observed in draining inguinal and axillary LNs and to a lesser extent in liver and 
spleen but not brachial, popliteal, mesenteric LNs, or brain (Figure 1D and Supplemental Figure 1; supplemental material available online with this article; https://doi.org/10.1172/jci.insight.126025DS1). In contrast, after i.v. delivery, liposomes were taken up at low levels in multiple LNs and brain and at much higher levels in liver and spleen (Supplemental Figure 1). When the biodistribution of dual ${ }^{3} \mathrm{H}$-calcitriol- and ${ }^{14} \mathrm{C}$-lipid-radiolabeled liposomes was tracked for 24 hours after s.c. delivery, most of the lipid and calcitriol accumulated at the skin injection site, but a small fraction of each rapidly dispersed to dLNs and blood and subsequently to spleen, liver, and kidney (Supplemental Figure 2, note that ${ }^{14} \mathrm{C}$-lipid provided a lower median percentage dose/g relative to ${ }^{3} \mathrm{H}$-calcitriol; ${ }^{14} \mathrm{C}$-DPPC is susceptible to exchange with the lipoprotein pool [ref. 35]). These data indicate that after s.c. injection, a proportion of the liposome dose rapidly reaches the $\mathrm{dLN}$ and additional LNs linked by lymphatic drainage. A further proportion enters the blood and can be taken up systemically, consistent with the intimate relationship between afferent and efferent lymphatics and the skin vasculature (25).

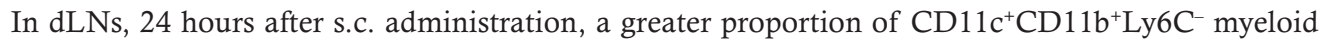
DCs and $\mathrm{CD} 11 \mathrm{c}^{+} \mathrm{CD} 11 \mathrm{~b}^{+} \mathrm{Ly} 6 \mathrm{C}^{+}$moDCs had taken up liposomes than $\mathrm{CD} 11 \mathrm{c}^{+} \mathrm{CD} 11 \mathrm{~b}^{-} \mathrm{CD} 8^{+} \mathrm{DCs}$, Siglec $\mathrm{H}^{+}$pDCs, or B cells in steady state and a greater proportion of moDCs had taken up liposomes than $\mathrm{CD}^{+} \mathrm{DCs}, \mathrm{pDCs}$, or $\mathrm{B}$ cells in the primed state. However, in the primed state, significantly higher proportions of $\mathrm{CD} 11 \mathrm{~b}^{+} \mathrm{Ly} 6 \mathrm{C}^{-}$and $\mathrm{Ly}_{6} \mathrm{C}^{+} \mathrm{DCs}$ had taken up peptide or peptide/calcitriol liposomes than had taken up blank liposomes, suggesting greater $\mathrm{dLN}$ retention or viability of migratory $\mathrm{CD} 11 \mathrm{~b}^{+} \mathrm{DCs}$ interacting with antigen-specific T cells (Figure 2A and Supplemental Figure 3). In the naive and primed states, $\mathrm{DiI}^{+} \mathrm{B}$ cells were more frequent than any other $\mathrm{DiI}^{+}$antigen-presenting cell (APC), whereas the $\mathrm{DiI}^{+} \mathrm{CD} 11 \mathrm{~b}^{-} \mathrm{DC}$ sere less frequent than any other $\mathrm{DiI}^{+} \mathrm{APC}$. DiI ${ }^{+} \mathrm{CD} 11 \mathrm{~b}^{+} \mathrm{Ly}_{6 c^{-}} \mathrm{DCs}$ were more frequent than $\mathrm{DiI}^{+} \mathrm{CD} 11 \mathrm{~b}^{+} \mathrm{Ly} 6 \mathrm{c}^{+} \mathrm{DC}$ in the steady state, while $\mathrm{DiI}^{+} \mathrm{CD} 11 \mathrm{~b}^{+} \mathrm{Ly} 6 \mathrm{c}^{+} \mathrm{DC}$ s were more frequent in the primed state (Figure 2A), consistent with evidence that moDCs expand in inflamed dLNs (27). Although fluorescein-labeled calcitriol was rapidly metabolized after injection, flow cytometric analysis confirmed that 5 hours after s.c. injection of dual-labeled liposomes, both lipid and calcitriol were colocated in dLN CD $11 b^{+}$DCs (Supplemental Figure 4).

After s.c. administration, soluble antigen or viral particles rapidly reach the subcapsular sinus, where they can be taken up by $\mathrm{CD} 11 \mathrm{~b}^{+} \mathrm{MHC}$ class $\mathrm{II}^{+} \mathrm{DCs}$ or macrophages adjacent to the fibroblast reticular cell (FRC) scaffold of the LN conduit system and subsequently transferred to B cells $(24,36-38)$. Given the rapid distribution of radiolabeled and DiI-labeled liposomes to dLNs, we ascertained traffic of liposomes incorporating TXR lipid after footpad administration to OVA/QuilA-primed mice. Fifteen minutes after administration, liposomes were observed in the subcapsular sinus of the popliteal LN (Figure 2B) and were concentrated at the entry of the afferent lymphatic, contacting podoplanin ${ }^{+}$FRC (yellow staining, Figure 2C). By 5 hours, liposomes were already visible deeper in the LN (arrows Figure 2, D and E), the intense red staining had disappeared, and discreet liposomes were observed in contact with $\mathrm{CD} 11 \mathrm{c}^{+} \mathrm{MHC}$ class $\mathrm{II}^{+}$ DCs (arrows) and the FRC in the paracortex (arrows), within and adjacent to CD19 ${ }^{+} \mathrm{B}$ cell follicles (Figure 2, F-I). Thus, after s.c. administration, liposomes rapidly access myeloid DCs and inflammatory moDCs in dLNs via afferent lymphatics and access LN FRC networks and the T-B junction. Within 24 hours, large number of B cells also contained liposomes.

Dose-dependent induction of antigen-specific peripheral Tregs and suppression of memory $T$ cells. To determine the threshold at which encapsulated peptide could trigger a proliferative response from naive antigen-specific $\mathrm{T}$ cells, we formulated liposomes with $400 \mathrm{ng} / \mathrm{ml}$ calcitriol and varying concentrations of $\mathrm{OVA}_{323-339^{\circ}}$ Three days after s.c. injection of $100 \mu \mathrm{l}$ of each formulation, the proliferative response of CTV-labeled naive splenic TCR-transgenic OVA-specific DO11.10 T cells was assessed. Liposome encapsulation of at least $10 \mu \mathrm{g} / \mathrm{ml}$ peptide (peptide dose $1 \mu \mathrm{g}$ ) was required for T cell proliferation (Supplemental Figure 5A). To determine the effect of the dose of encapsulated peptide on peripheral Treg ( $p$ Treg) induction, each formulation was injected s.c. twice, 1 week apart, to DO11.10 mice, and then Foxp3 ${ }^{+}$pTregs were assessed in peripheral blood (PB) on day 10 and spleen on day 18 . Foxp $3^{+}$pTregs significantly increased in $\mathrm{PB}$ and spleen in response to liposomes encapsulating $400 \mathrm{ng} / \mathrm{mL}(0.96 \mu \mathrm{M})$ calcitriol and $30 \mu \mathrm{g} / \mathrm{mL}(16.9 \mu \mathrm{M})$ OVA $_{323-339}$ (Supplemental Figure 5B). One day after injection of 0-90 $\mu \mathrm{L}$ DiI-labeled calcitriol/OVA ${ }_{323-339}$ liposomes to primed BALB/c mice, uptake by $C D 11 c^{+} C D 11 b^{+}$DCs was proportional to the delivered volume (Supplemental Figure $5 \mathrm{C}$ ). Furthermore, after 2 injections of liposomes encapsulating $400 \mathrm{ng} / \mathrm{mL}$ calcitriol and $30 \mu \mathrm{g} / \mathrm{mL} \mathrm{OVA}_{323-339}$ to DO11.10 mice, splenic Foxp $3^{+}$pTregs significantly increased in response to $100 \mu \mathrm{L}$ but not $30 \mu \mathrm{L}$ liposomes (Supplemental Figure 5C). These data demonstrate that induction of antigen-specific pTregs is antigen dose dependent and define the liposomal peptide concentration and volume of administration for subsequent experiments. 
A

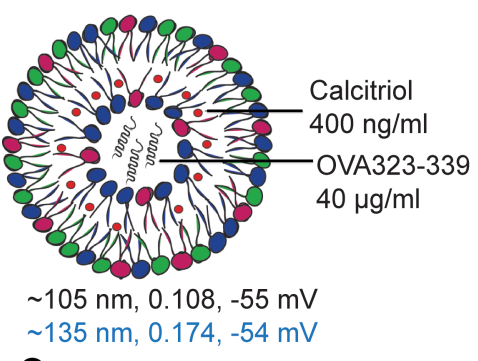

C

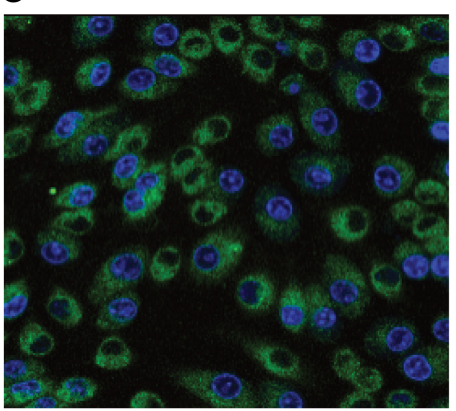

BODIPY-FL-calcitriol

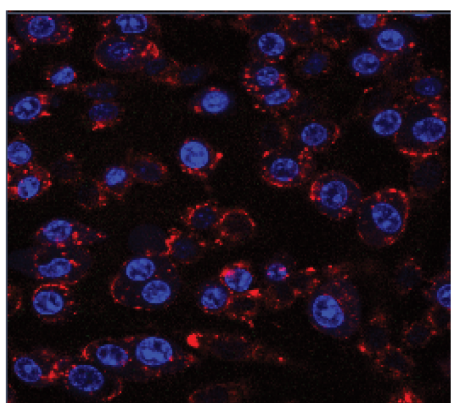

Texas Red lipid

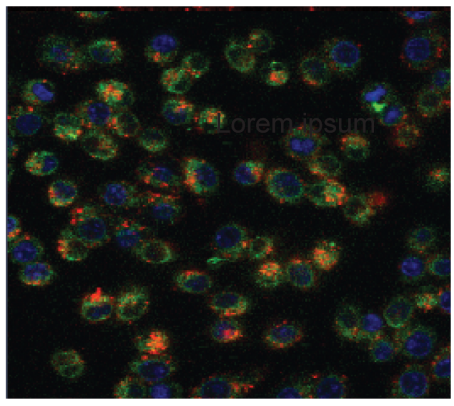

Merge
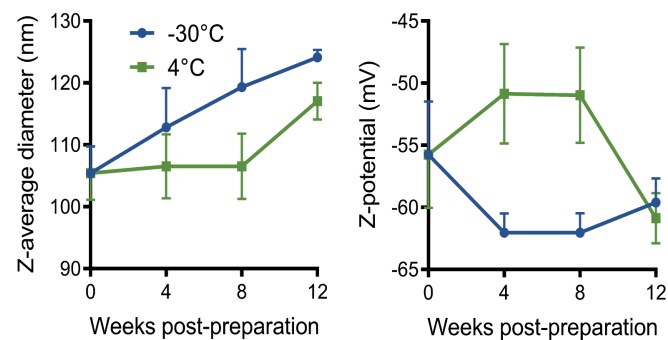

B

Texas red-labeled lipid

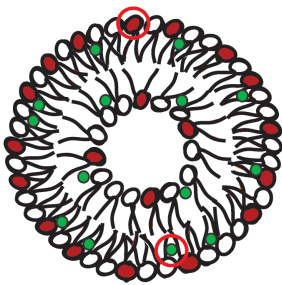

BODIPY-FL-labeled calcitriol

D

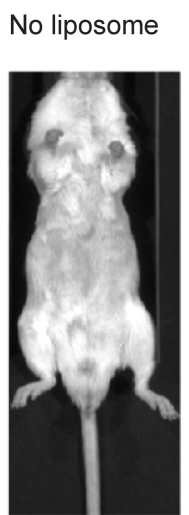

S.C. liposome

OVA/QuilA

s.c. liposome
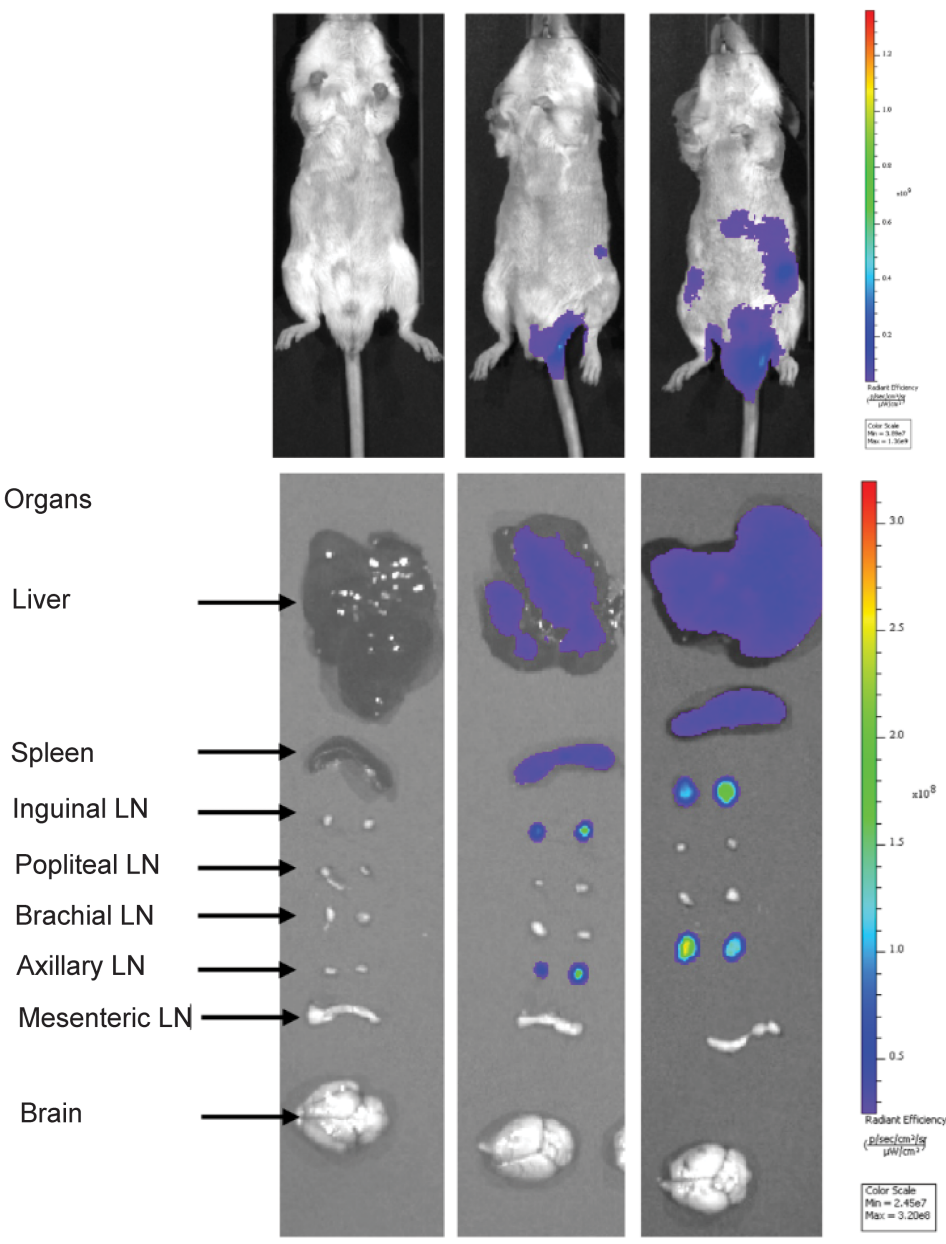

Figure 1. Liposome characteristics and biodistribution. (A) Size, polydispersity (PDI), and surface charge (black: thin film hydration method, blue: microfluidic method) of liposomes encapsulating calcitriol and $\mathrm{OVA}_{323-339}$ maintained in $\mathrm{PBS}$ at $-30^{\circ} \mathrm{C}, 4^{\circ} \mathrm{C}$, or $25^{\circ} \mathrm{C}$ for 1 month; $n=3$ experiments. (B and $\mathbf{C}$ ) Single- or dual-labeled liposomes were incubated with RAW 264.7 macrophages and uptake analyzed after 2 hours by confocal microscopy (original magnification, $\times 60$ ). Blue: DAPI, Green: FL-calcitriol, Red: TXR-lipid. (D) DiR-labeled OVA ${ }_{323-339} /$ calcitriol liposomes were injected s.c. into the tail base of naive or mice primed at tail base with OVA/QuilA 3 days previously. Mice and tissues were analyzed 24 hours after liposome injection by IVIS in vivo imaging. (C and D) Representative of $n=3$ per group.

To define the effect of liposomal calcitriol in the steady state, DO11.10 $\mathrm{T}$ cells were transferred to $\mathrm{BALB} / \mathrm{c}$ mice, and $100 \mu \mathrm{L}$ liposomes encapsulating $40 \mu \mathrm{g} / \mathrm{mL} \mathrm{OVA}_{323-339}$ with or without $400 \mathrm{ng} / \mathrm{mL}$ calcitriol were administered s.c. twice. $\mathrm{CD} 4^{+} \mathrm{Foxp}^{+}$pTregs significantly increased within 6 days of a single injection of calcitriol/OVA but not OVA liposomes and increased further after the second injection. Similarly, calcitriol was required for the upregulation of CD73, a marker of $\mathrm{T}$ cell anergy (39), by residual 
A

Blank liposomes

OVA liposomes

OVA/calcitriol liposomes
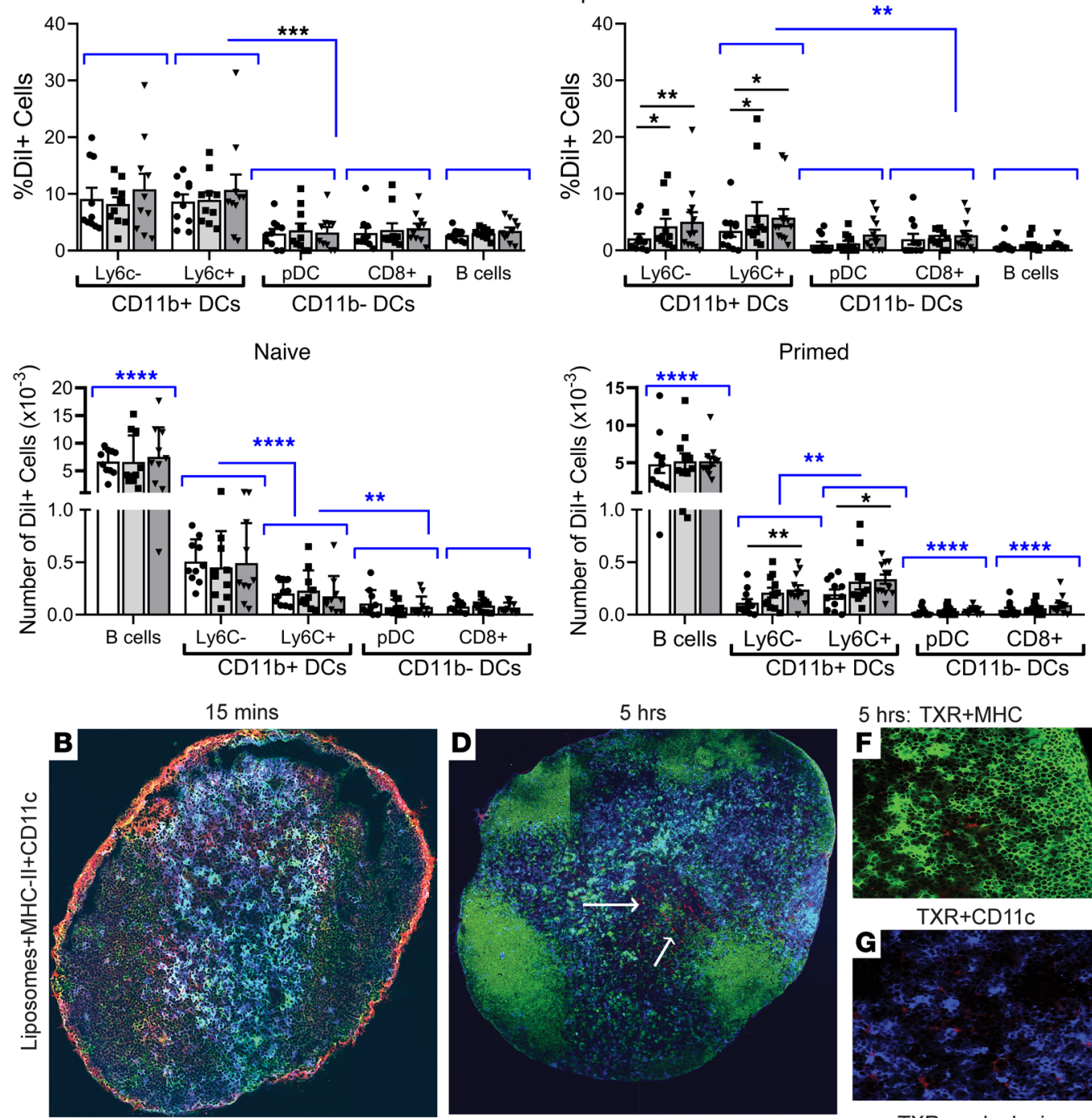

5 hrs: TXR+MHC
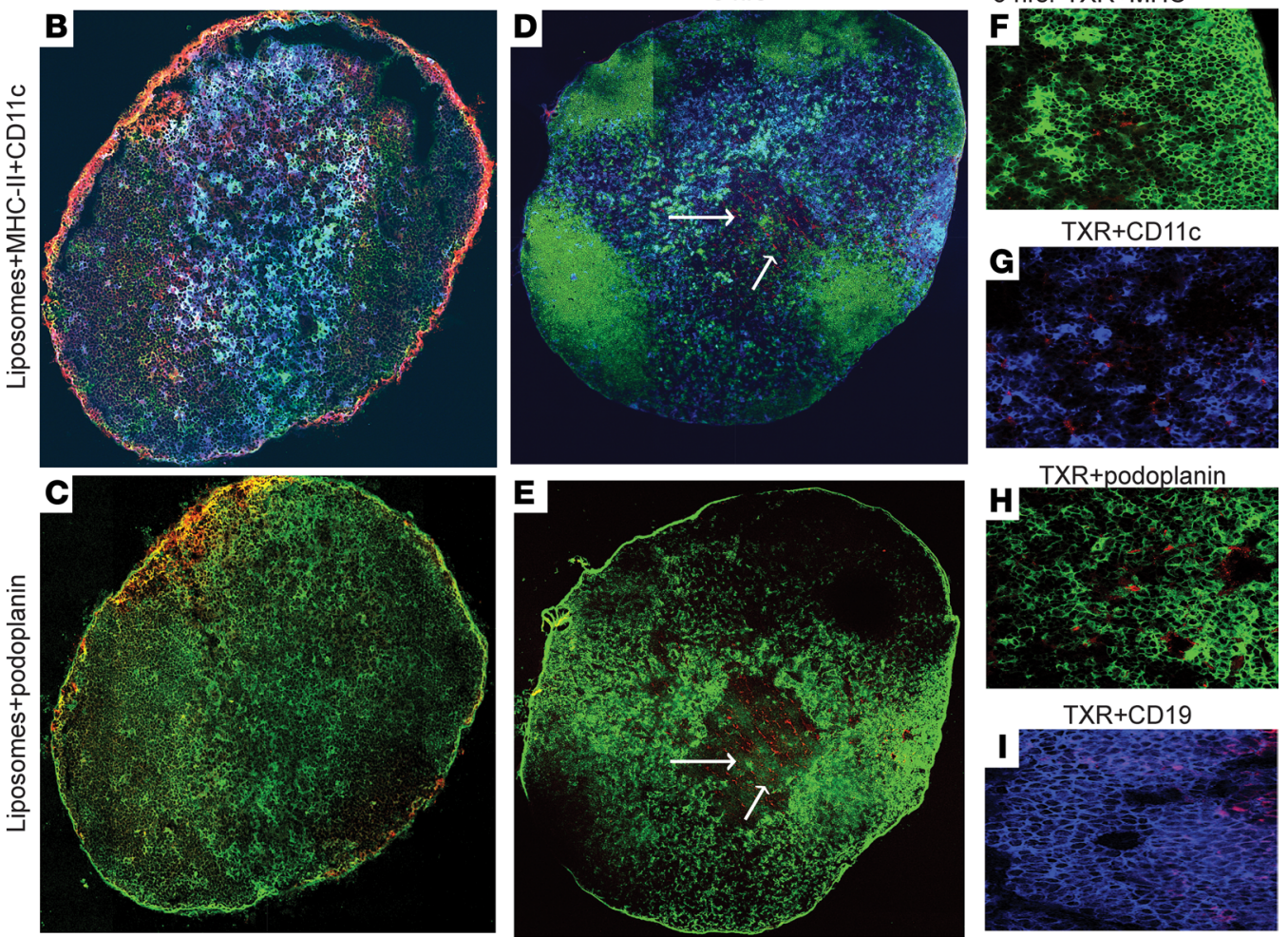

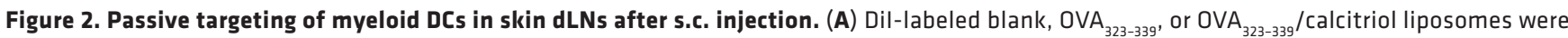
injected as in Figure 1D, inguinal lymph node (ILN) cells were stained with CD11c, CD11b, MHC class II, Siglec H, Ly6C, and CD19. Dil+ populations were gated as shown in Supplemental Figure 1A. Total number of Dil' cells. Flow cytometric data pooled from 10-12 mice/group, representing 2 replicates. ${ }^{*} P<0.05 ;{ }^{* *} P<0.01 ;{ }^{* *} P<0.001 ;{ }^{* * *} P<0.0001$. Analyses shown in blue are for each cell type, uptake of pooled blank, OVA, and OVA/calcitriol liposomes. (B-I) Popliteal lymph nodes from mice primed with OVA/QuilA 3 days before were removed 15 minutes and 5 hours after footpad injection of $\mathrm{OVA}_{323-339} /$ calcitriol liposomes incorporating Texas red-DPPC (red) lipid. Lymph nodes were stained with MHC class II-FITC (green) (B, D, and F), CD11C-APC (blue) (B, D, and G), podoplanin-FITC (green, FRC) (C, E, and H), and CD19 (blue) (I). Original magnification, $\times 20$ [B-E, tiled images]; $\times 20$ [F-I, representative of 3 experiments]). Differences in proportion of Dil' cells compared by mixed-effects analysis with Geisser-Greenhouse correction and Holm-Sidak's multiple comparison test. 
Foxp3- T cells. The proportion of Foxp3-CXCR5- OVA-specific T cells expressing the PD1 marker of antigen experience and the intensity of PD1 expression increased in response to OVA liposomes and to an even greater extent after OVA/calcitriol liposomes. PD1 and CD73 were coexpressed by Foxp3- $\mathrm{T}$ cells (Figure 3A and Supplemental Figure 6). These data indicate that in a noninflammatory setting, peptide/ calcitriol liposomes induce antigen-specific Tregs and promote PD1 expression and anergy. To dissect the role of each component of the formulation, we administered blank liposomes, OVA/calcitriol liposomes, or equivalent doses of nonencapsulated OVA and calcitriol twice after DO11.10 T cell transfer. While the proportion of OVA-specific Foxp3 increased with liposomal or nonliposomal delivery, expression of the effector Treg marker ICOS by Tregs, PD1 by Foxp3- $\mathrm{T}$ cells, and suppression of IFN- $\gamma$ production required liposomal codelivery of antigen and calcitriol (Figure 3B). Since there was no evidence of PD1 expression (i.e., antigen experience) after nonliposomal delivery, these data suggest direct effects of calcitriol on the TCR-transgenic T cells. Direct T cell effects of vitamin D3 were noted previously, due to enhanced TGF- $\beta$ bioactivity (40). Furthermore, differentiation of antigen-specific effector Tregs and development of anergy require antigen experience.

To determine which targeted APCs are required for Treg induction, we administered OVA/QuilA and liposomes to BALB/c mice, sorted dLN APC populations 24 hours later, and then incubated them with DO11.10 T cells in the presence of IL-2. OVA-specific Tregs were induced in response to DCs but not B cells or FRC sorted from mice administered OVA/calcitriol liposomes (Figure 3C).

To investigate whether peptide/calcitriol liposomes can induce antigen-specific $\mathrm{T}$ cell tolerance in an inflammatory setting, DO11.10 T cells were transferred into BALB/c hosts. After s.c. administration of $100 \mu \mathrm{L}$ liposomes encapsulating $400 \mathrm{ng} / \mathrm{mL}$ calcitriol and $30 \mu \mathrm{g} / \mathrm{mL} \mathrm{OVA}_{323-339}$ twice and concomitant immunization with OVA/QuilA, fewer OVA-specific T cells were recovered from the spleen and more of these were Tregs than in mice administered PBS. Moreover, residual cells were anergic, as fewer produced IFN- $\gamma$ after restimulation ex vivo with OVA $_{323-339}$ (Figure 3D).

In autoimmune inflammatory disease, antigen-specific effector memory $\mathrm{T}$ cells (Tem cells) may be primed long before disease develops. To test whether peptide/calcitriol liposomes could control antigen-specific memory $\mathrm{T}$ cells in an inflammatory setting, DO11.10 T cells were incubated ex vivo with antigen and cytokine. Ex vivo-generated OVA-specific memory T cells $\left(40 \% \mathrm{CD} 44^{\text {hi }} \mathrm{CD} 62 \mathrm{~L}^{\mathrm{lo}}, 56 \%\right.$ CD $44^{\text {hi }} \mathrm{CD} 62 \mathrm{~L}^{\mathrm{hi}}$, Supplemental Figure 7) were transferred to BALB/c mice. Expansion of these OVA-specific memory T cells decreased and Foxp3 expression increased in response to liposomes encapsulating calcitriol and 30 $\mu \mathrm{g} / \mathrm{mL} \mathrm{OVA}_{323-339}$, but not $30 \mu \mathrm{g} / \mathrm{mL}$ irrelevant $\mathrm{HA}_{126-138}$ peptide (Figure $3 \mathrm{E}$ ), demonstrating antigen specific memory $\mathrm{T}$ cell control. We further explored the persistence of OVA-specific regulation of inflammatory responses by concomitant OVA immunization and administration of blank or OVA/calcitriol liposomes twice, followed by analysis of OVA-specific immunity and Foxp3 ${ }^{+}$Tregs 5 and 42 days later in response to OVA challenge (Figure 3F). Five days after the second administration of OVA/calcitriol liposomes, OVA-specific T cells and IFN- $\gamma^{+} \mathrm{T}$ cells were reduced, while Foxp3 ${ }^{+}$Treg and Foxp3-PD1 ${ }^{+} \mathrm{T}$ cells increased. By day 42, OVA-specific T cells failed to reexpand or to produce IFN- $\gamma$ and Tregs were still increased relative to mice receiving empty liposomes. These data demonstrate persistence of antigen-specific memory pTregs for more than 1 month and prolonged antigen-specific unresponsiveness induced by OVA/calcitriol liposomes.

To determine the functional effects of liposome-induced OVA-specific pTregs, we adoptively transferred a cohort of DO11.10 T cells and treated recipients with s.c. OVA/calcitriol liposomes, before transferring a second cohort of labeled DO11.10 T cells and immunizing with OVA/QuilA. In the first T cell cohort, OVA-specific T cells failed to expand, their capacity for IFN- $\gamma$ production decreased, and Foxp $3^{+}$ pTregs increased in response to OVA/calcitriol liposomes. The T cell proliferative and IFN- $\gamma$ responses of the second $\mathrm{T}$ cell cohort decreased relative to mice treated with blank liposomes. Furthermore, both IL-10 production and Foxp3 expression increased in the second T cell cohort (Figure 3G). These data are consistent with an "infectious" process of tolerance whereby calcitriol-mediated tolerogenic effects in targeted DCs induce antigen-specific pTregs, which then control antigen-specific Teffs after reexposure to their cognate antigen under inflammatory conditions, promoting their production of IL-10.

Uptake by $P D-L 1^{\text {hi }} C D 11 b^{+} D C S$ and $P D-L 1$-dependent antigen-specific regulation. In inflammatory autoimmune diseases such as RA, activated DCs present autoantigens to Teffs under immune-stimulatory conditions, mediated through cytokine- and neutrophil-mediated signals and microbiota-derived TLR agonists (41-43). To explore tolerogenic mechanisms used by inflammatory DCs, we assessed MHC class II, 
A $\square$ Blank Liposomes

$\square$ OVA-Calcitriol Liposomes
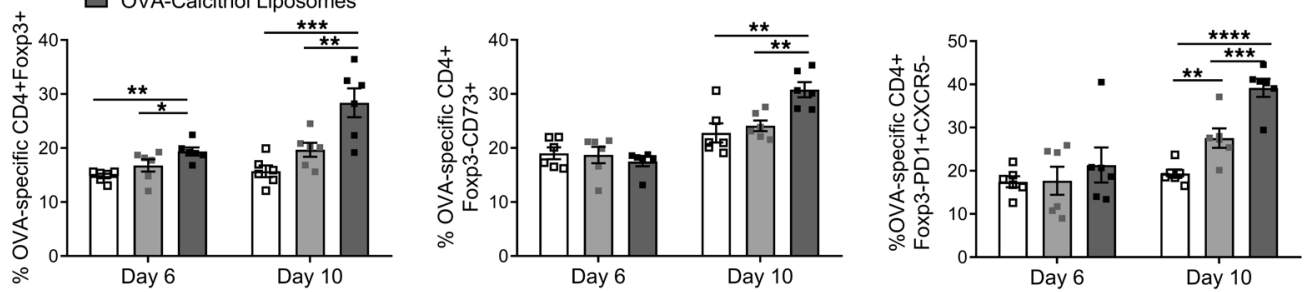

B
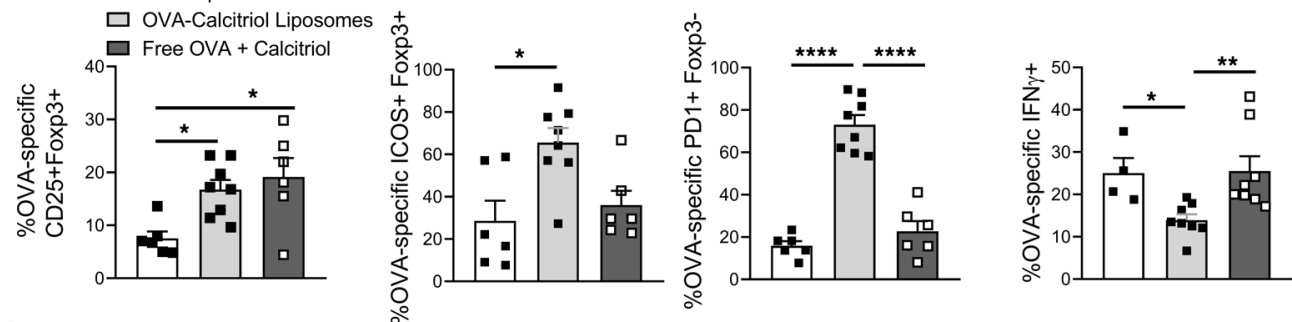

C

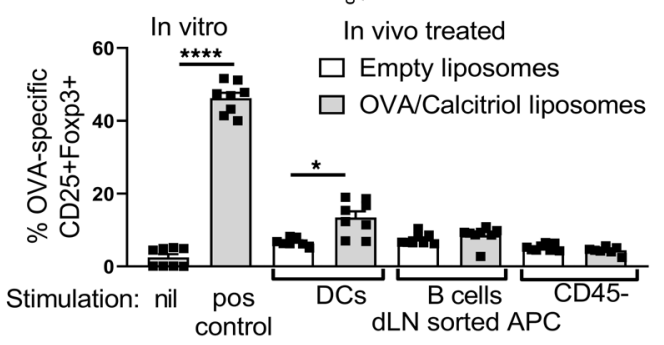

D $\square$ PBS

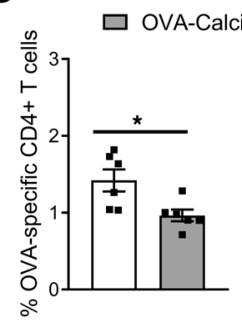

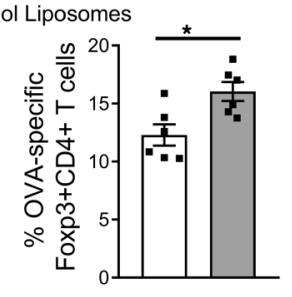

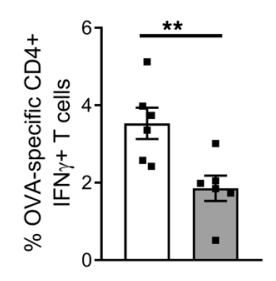

$\mathbf{E}$

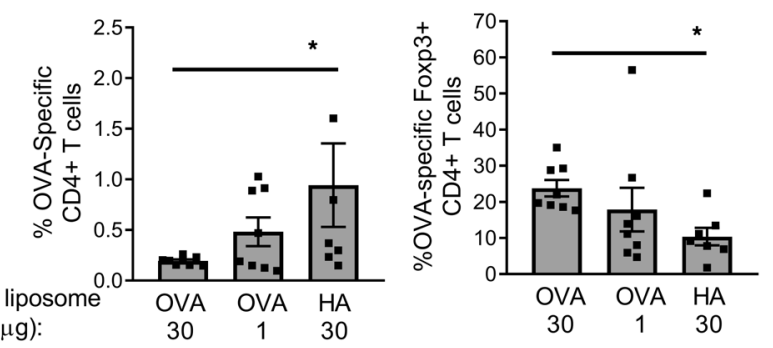

$\mathbf{F}$

$\square$ Empty liposomes peptide $(\mu \mathrm{g})$ :

$\square$ OVA-calcitriol liposomes

G
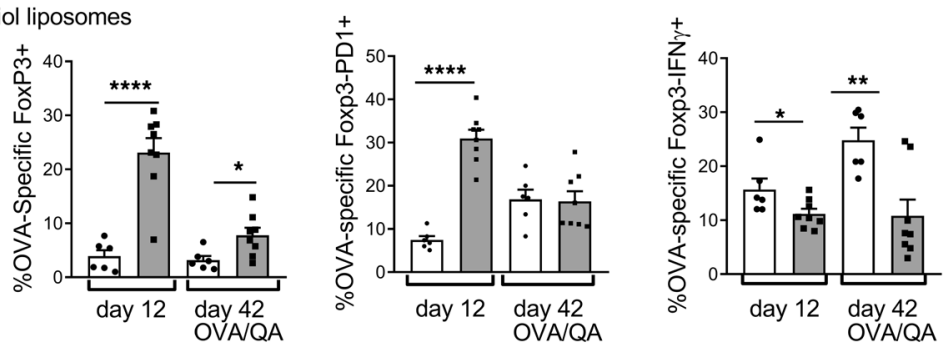

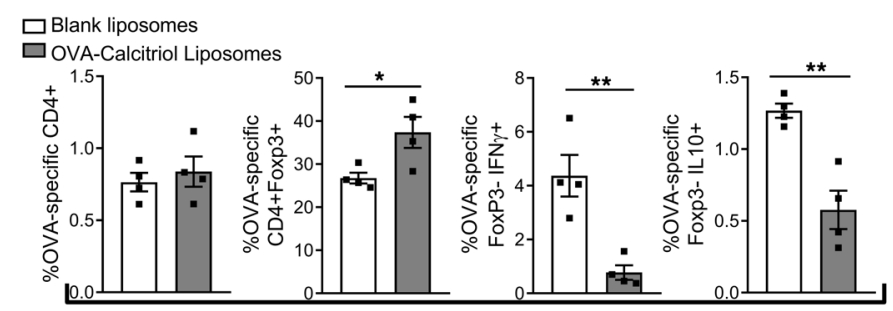

OVA-specific cohort 1

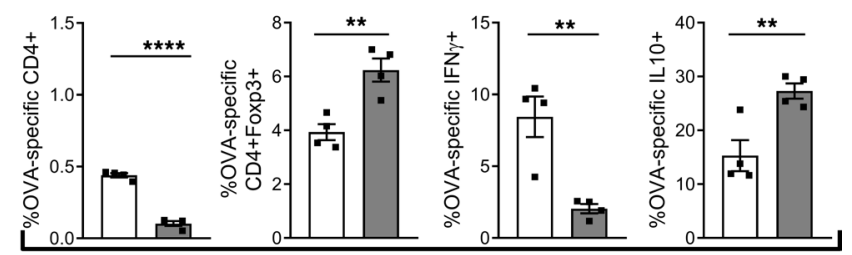

OVA-specific cohort 2 
Figure 3. Induction of antigen-specific pTregs, prolonged suppression of antigen-specific immunity, and infectious tolerance after s.c. injection of calcitriol/OVA ${ }_{323-339}$ liposomes. (A) D011.10 T cells were transferred to BALB/c hosts. Mice were administered liposomes s.c. once on day 0 or twice on days 0 and 6 . Splenocytes were analyzed on day 6 ( 1 injection) or 10 ( 2 injections). (B) After adoptive transfer of D011.10 T cells, mice were administered liposomes or the same concentrations of nonencapsulated $\mathrm{OVA}_{323-339}$ and calcitriol s.c. on days 0 and 6; splenocytes were analyzed on day 12 . For intracellular (i.c.) IFN- $\gamma$ analysis, splenocytes were restimulated overnight with $\mathrm{OVA}_{323-339^{\circ}}$ (C) Thee days after s.c OVA/QuilA priming, liposomes were administered s.c. After 24 hours, sorted dLN MHCII+CD11 $\mathrm{C}^{+} \mathrm{DCs}$, MHCII+CD19+ $\mathrm{B}$ cells, and CD45- stromal cells were incubated with D011.10 CD4 ${ }^{+} \mathrm{T}$ cells in the presence of IL-2. In vitro positive control D011.10 CD4 ${ }^{+}$T cells were stimulated with anti-CD3 and anti-CD28 in presence of IL-2, TGF- $\beta$, anti-IL-4, and anti-IFN- $\gamma$. CD25 ${ }^{+}$Oxp $^{+}$Tregs were enumerated 5 days later. (D) After D011.10 T cell transfer, mice were immunized with OVA/QuilA (day 0). Liposomes were administered s.c. on days 0 and 7. Splenocytes were analyzed on day 12 and after overnight $\mathrm{OVA}_{323-339}$ stimulation for i.c. IFN- $\gamma$. (E) $5 \times 10^{6} \mathrm{DO} 11.10$ in vitro-generated memory T cells were adoptively transferred to BALB/c hosts, which were administered liposomes s.c. on days 0 and 7. Mice were immunized with OVA/QuilA on day 10 and splenocytes were analyzed on day 17. (F) After D011.10 T cell transfer, mice were primed with OVA/QuilA

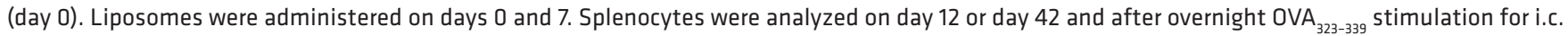
IFN- $\gamma$. Mice analyzed later were boosted with OVA/QuilA on day 35. (G) After adoptive transfer of $5 \times 10^{6} \mathrm{DO11.10}$ T cells, BALB/C hosts were administered calcitriol/OVA ${ }_{323-339}$ liposomes twice s.c. into the tail base, 1 week apart. A second cohort of $5 \times 10^{6}$ CFSE-labeled DO11.10 T cells was adoptively transferred, and mice were immunized with OVA/QuilA on day 10. Splenocytes were analyzed on day 15. For i.c. IFN- $\gamma$ and IL-10 analysis, splenocytes were restimulated overnight with $\mathrm{OVA}_{323-339^{\circ}} n=6-8$ per group, representative of 2 replicates. ${ }^{*} P<0.05 ;{ }^{* *} P<0.01 ;{ }^{* * *} P<0.001 ;{ }^{* * * *} P<0.0001$. ANOVA with Tukey's multiple comparison test (A-C and $\mathbf{E})$ and $t$ tests $(\mathbf{D}, \mathbf{F}$, and $\mathbf{G})$.

costimulatory, and coinhibitory molecules expressed by dLN DCs targeted by liposomes under resting or inflammatory conditions. We injected DiI-labeled liposomes containing no payload, OVA, or OVA/calcitriol s.c. to naive BALB/c mice or mice primed with OVA/QuilA 3 days previously. CD11b ${ }^{+} \mathrm{Ly}^{6} \mathrm{C}^{-} \mathrm{DiI}^{+}$ DCs phagocytizing liposomes, irrespective of payload, expressed significantly higher levels of PD-L1 than DiI- DCs under primed conditions, and PD-L1 was expressed at significantly higher levels in DiI ${ }^{+}$DCs in the primed state than in the steady state (Figure 4A). Notably, PD-L1 ${ }^{+} \mathrm{MHC}$ class $\mathrm{II}^{+}$cells (arrowheads, likely DCs) and PD-L1 ${ }^{+} \mathrm{MHC}$ class $\mathrm{II}^{-}$cells (white arrow, likely FRC) were observed predominantly in paracortex and adjacent to the afferent lymphatic (yellow arrow) of primed LNs (Figure 4B). In contrast, in naive LNs, PD-L1 was barely expressed (Figure 4C). Together, these data indicate that, in the primed setting, CD11b ${ }^{+}$DCs preferentially taking up s.c.-administered liposomes express high levels of PD-L1.

In contrast to PD-L1, after OVA immunization, MHC class II expression specifically increased on the

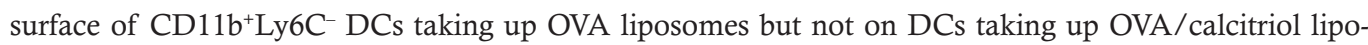
somes (Figure 4D). Thus, when primed T cells are present for DC cross-talk, the increase in MHC class II expression that occurs with targeted delivery of cognate peptide is suppressed by calcitriol codelivery.

To assess the role of PD-L1 in antigen-specific tolerance, we treated naive or OVA/QuilA-primed mice with OVA/calcitriol liposomes along with anti-PD-L1 or isotype control $\mathrm{mAb}$. In the draining LNs of naive mice administered OVA/calcitriol liposomes, OVA-specific T cells bearing the PD1 marker of antigen-experience expanded, independent of $\mathrm{PD}-\mathrm{L} 1$. In the spleen, $\mathrm{PD} 1^{+} \mathrm{OVA}-$ specific $\mathrm{T}$ cell expansion was controlled and Tregs increased in a PD-L1-dependent manner (Figure 5A). When previously primed, expansion of $\mathrm{PD} 1^{+}$OVA-specific T cells was suppressed in draining LNs and Tregs were increased. This increase was again PD-L1-dependent (Figure 5A). In support, after 2 s.c. administrations of OVA/calcitriol liposomes to naive mice, overall CD11 $\mathrm{b}^{+}$Ly6 $\mathrm{C}^{-}$DC PD-L1 expression was higher in spleen than $\mathrm{dLN}$. Thus, liposomes targeting antigen and calcitriol to DCs promote PD-L1-dependent antigen-specific T cell regulation either locally or at distant PD-L1-expressing sites (Figure 5B). Together, these data indicate that, in immunized individuals, calcitriol modulates APC function of $\mathrm{CD} 11 \mathrm{~b}^{+} \mathrm{Ly} 6 \mathrm{C}^{-} \mathrm{dLN}$ DCs targeted by peptide/calcitriol liposomes, leveraging adjuvant-induced PD-L1 expression and suppressing MHC class II-dependent antigen presentation in the context of $\mathrm{T}$ cell-DC cross-talk.

To further explore this in context of infectious tolerance, we repeated the 2-cohort experiment in mice receiving either anti-PD-L1 or isotype control when treated with liposomes. With isotype treatment, OVA-specific Treg and IFN- $\gamma$ anergy were induced in the spleens of both cohorts, while IL-10 increased only in cohort 2. The effect of liposomes on Tregs and IL-10 was PD-L1 dependent, while the effect of liposomes on IFN- $\gamma$ anergy was not (Figure 5C). However, PD-L1 inhibition did reduce the OVA-specific IFN- $\gamma$ response to $\mathrm{OVA}_{323-339}$ stimulation in control mice, likely due to PD-L1 tuning of DC-T cell antigen responsiveness, as previously described (44). Since both OVA-specific Tregs and IL-10 were suppressed in OVA/calcitriol liposome- and PD-L1-treated mice, the reduction in IFN- $\gamma$ is more likely to be due to deletion, with unresponsive residual $\mathrm{T}$ cells, than regulation.

Calcitriol liposomes modulate human DC phenotype and function. To assess the effect of liposomes on human cells under inflammatory conditions, we incubated PB mononuclear cells (PBMCs) with CpG 
A

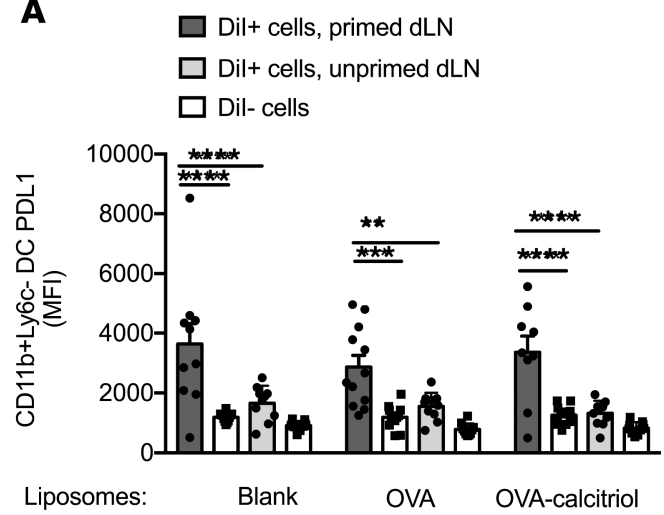

B

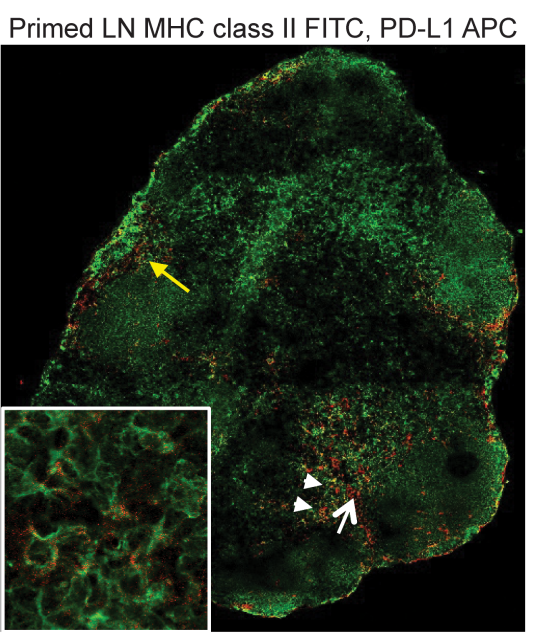

c

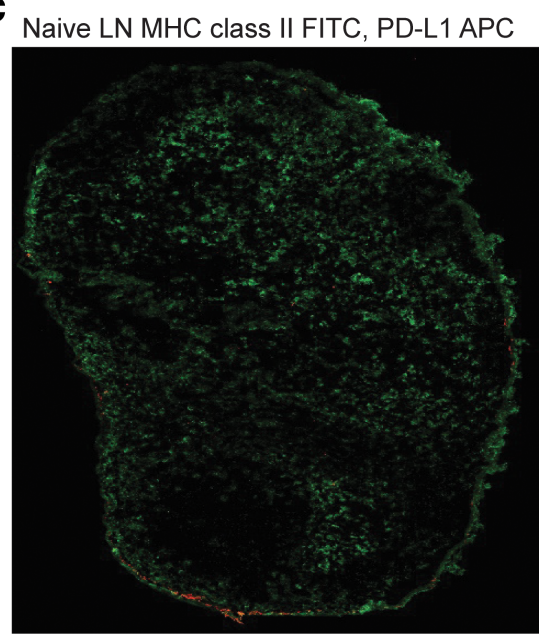

Figure 4. Calcitriol-antigen liposomes, taken up by PD-L1hi CD11b+ primed DCs, suppress DC MHC class II expression. (A) BALB/C mice were immunized with OVA/QuilA s.c. or were left unprimed and then 3 days later were injected s.c. (both injections at the tail base) with Dil-labeled liposomes. Mean fluorescence intensity (MFI) of $\mathrm{PD}-\mathrm{L} 1$ in gated $\mathrm{Dil}^{+}$and $\mathrm{Dil}^{-} \mathrm{dLN}$ CD11 b ${ }^{+}$Ly6C- DCs 24 hours later. (B) Popliteal lymph node (original magnification, $\times 10 ; \times 40$ [inset]) from a mouse primed with OVA/QuilA 3 days previously was stained with MHC class II-FITC (green) and PD-L1APC (red) (yellow arrow, afferent lymphatic entry; white arrow, PD-L1+MHC class II- cells; white arrowheads, PD1+MHC class II+ cells.). (C) Poplitea lymph node (original magnification, $\times 10$, relative to isotype control) from a naive mouse stained with MHC class II-FITC (green) and PD-L1-APC (red). Images in $\mathbf{B}$ and $\mathbf{C}$ are representative of 2 replicates. (D) Mice were treated as in $\mathbf{A}$. MFI of MHC class II in gated Dil ${ }^{+}$and Dil- dLN CD11b+Ly6C $D C s 24$ hours later. $n=10-12$ per group, representative of 2 replicates. ${ }^{*} P<0.05 ;{ }^{*} P<0.01 ;{ }^{* *} P$ $<0.001$; ${ }^{* * *} P<0.0001$, ANOVA with Sidak's multiple comparison test.

D $\square$ Dil+ cells, primed dLN

$\square$ Dil+ cells, unprimed dLN

$\square$ Dil- cells

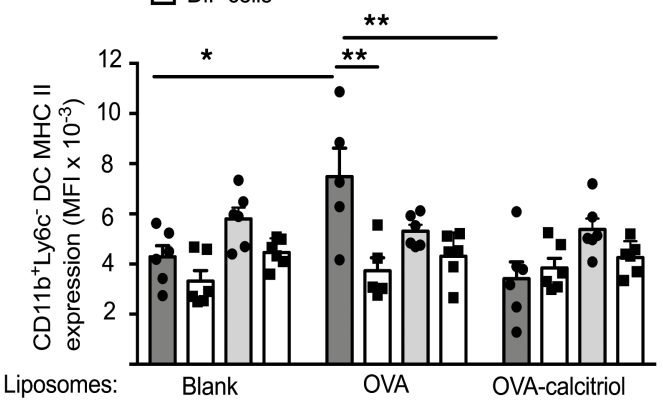

oligonucleotide in the presence of blank liposomes, calcitriol liposomes, or equivalent concentrations of free calcitriol. Calcitriol liposomes were phagocytized by $90 \%-100 \%$ CD $1 c^{+}$DCs within 1 hour in vitro. After exposure to calcitriol liposomes or free calcitriol, secretion of TNF, IL-6, IL-10, and IFN- $\alpha$ was dose-dependently reduced, with greater suppression when calcitriol was liposome encapsulated (Figure 6A). These data are consistent with NF-кB inhibition by calcitriol and underline the inflammatory suppression associated with targeting of calcitriol to phagocytic cells. To assess moDC phenotype and function, we incubated them with calcitriol liposomes and then stimulated them with LPS or allogeneic T cells. Calcitriol liposome-treated moDCs took up liposomes readily (Figure 6B), expressed lower levels of CD40 than untreated or blank liposome-treated moDCs in the presence of LPS (Figure 6C), and promoted greater Treg differentiation in allogeneic mixed lymphocyte cultures (Figure 6D). Thus, in inflammatory settings, calcitriol liposome uptake by mouse and human myeloid DCs modifies antigen-presenting capacity, promoting Treg differentiation. 
A

Naive mice

Draining LN

$\mathrm{CD}^{+} \mathrm{T}$ cell

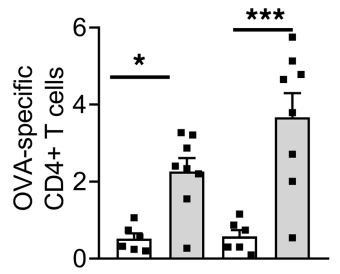

Foxp3+ Treg

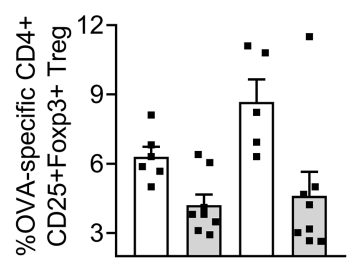

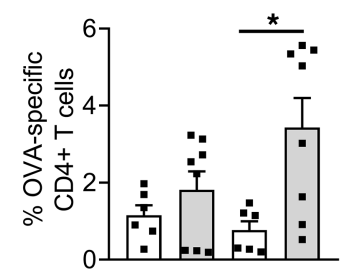

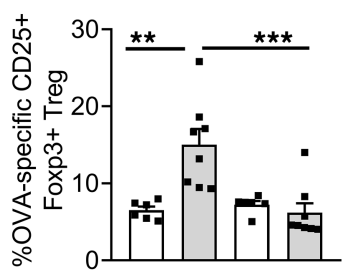

Primed draining LN

$\square$ Blank Liposomes

$\square$ OVA-Calcitriol Liposomes
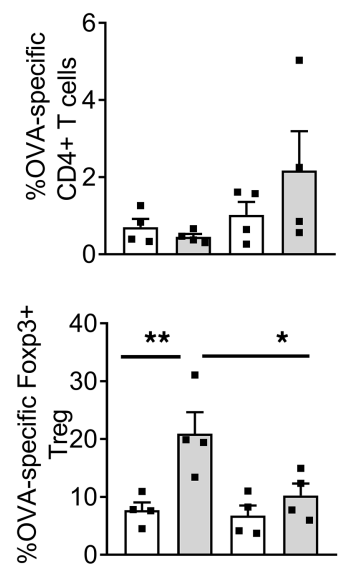

Foxp3-PD1+ T cells
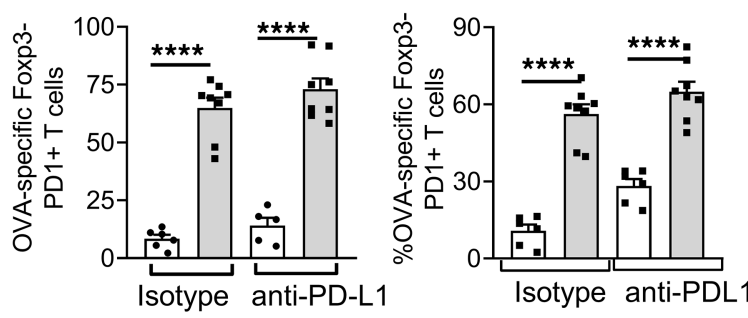

C

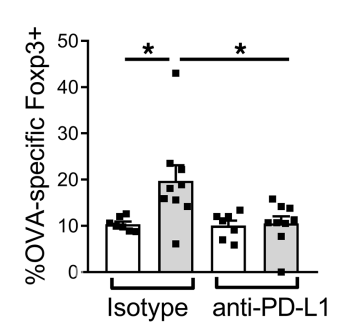

OVA-specific cohort 1

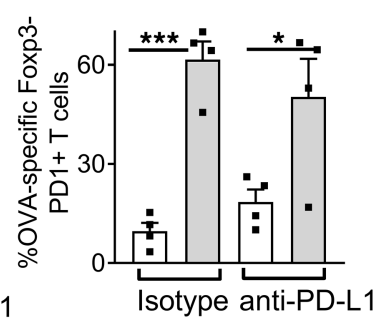

$\square$ Blank Liposomes

$\square$ OVA-Calcitriol Liposomes
B

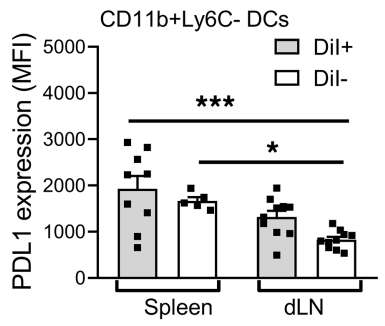

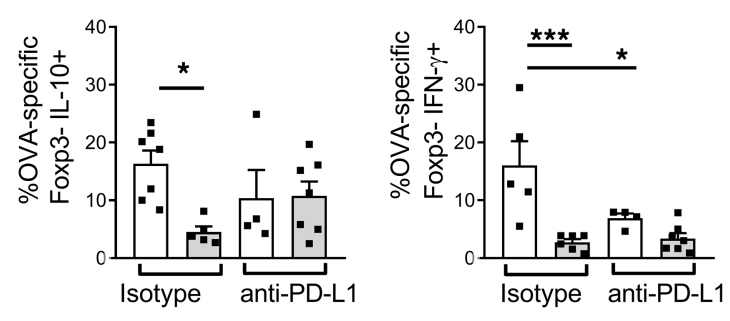

Figure 5. Calcitriol-antigen liposomes induce antigen-specific PD-L1-dependent tolerance. (A) BALB/c hosts received D011.10 T cells and either anti-PD-L1 or isotype mAb. Naive or OVA/ QuilA-primed mice (day 0 ) were administered calcitriol/OVA ${ }_{323-339}$ liposomes s.c. into the tail base on days 0 and 6 . ILN or splenocytes were analyzed by flow cytometry on day 10 (naive) or 12 (primed). (B) Experimental conditions as for $\mathbf{A}$ in naive mice: MFI of PD-L1 in gated $\mathrm{Dil}^{+}$and $\mathrm{Dil}^{-} \mathrm{dLN}$ and spleen CD11b+Ly6C- DCs 24 hours after the second liposome administration. (C) Experimental conditions were as in Figure 3G, except that groups of mice received either anti-PD-L1 or isotype mAb. $n=4-12$ per group, representative of 2 replicates. ${ }^{*} P<0.05$ ${ }^{* *} P<0.01 ;{ }^{* * *} P<0.001 ;{ }^{* * *} P<0.0001,2$-way ANOVA with Tukey's multiple comparison test.

Antigen-specific suppression of systemic inflammatory autoimmune disease models. Having demonstrated the

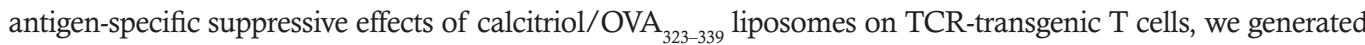
liposomes encapsulating disease-specific peptides and calcitriol to determine the response of endogenous autoreactive $\mathrm{T}$ cells and the disease response in models of RA and vasculitis. Proteoglycan-induced inflammatory arthritis (PGIA) is driven by autoreactivity toward the G1 antigenic region of human proteoglycan (aggrecan), a cartilage antigen with widespread expression where IA $^{\mathrm{d}}$-restricted aggrecan ${ }_{89-103}$ is the dominant $\mathrm{T}$ cell epitope $(45,46)$. When administered 7 and 14 days before initiation of disease, liposomes encapsulating both calcitriol and aggrecan ${ }_{89-103}$ but not aggrecan ${ }_{89-103}$ alone significantly suppressed disease severity, histological severity of ankle arthritis, and anti-hPG antibodies 56 days after PGIA initiation (Figure 7A). We assessed the response 


$$
\begin{aligned}
& \rightarrow \text { Blank liposomes + CpG } \\
& \text { - Calcitriol liposomes + CpG } \\
& \text { - Free calcitriol + CpG }
\end{aligned}
$$
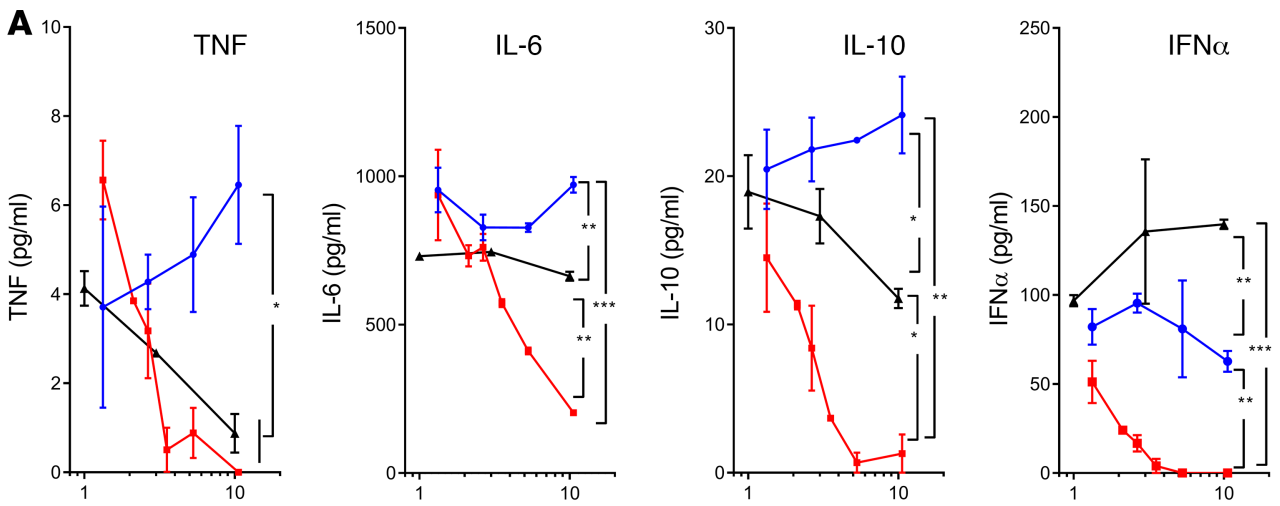

B

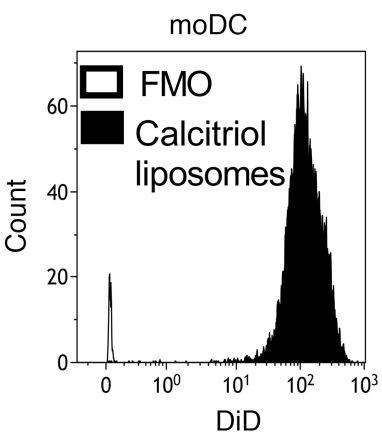

C
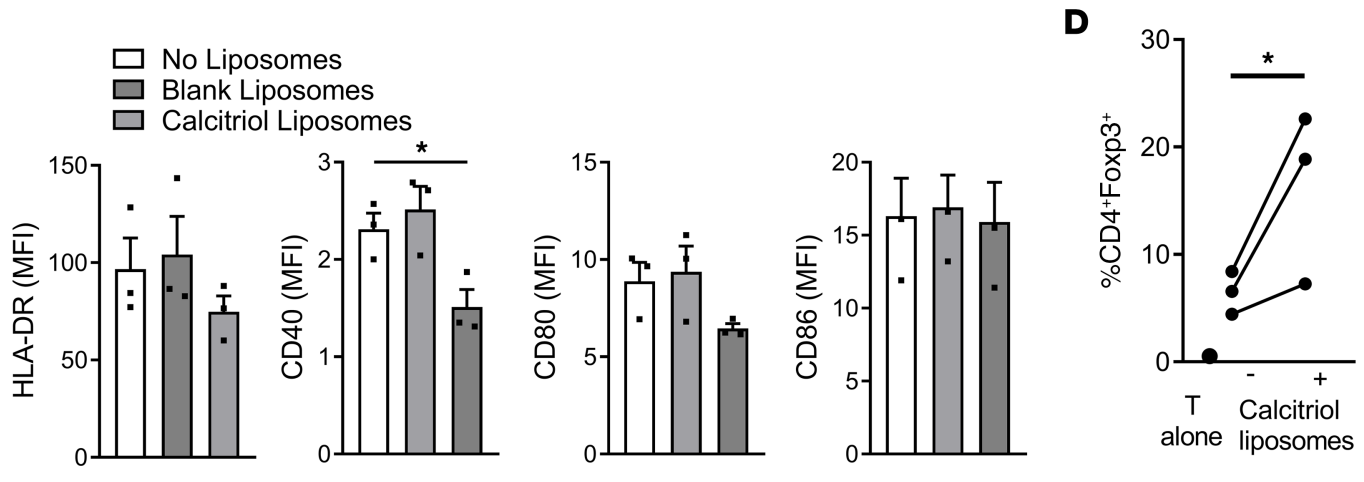

Figure 6. Calcitriol liposomes modulate human DC phenotype and function. (A) Human PBMCs were stimulated for 18 hours with $10 \mu \mathrm{g} / \mathrm{ml} \mathrm{CpG}$ in the absence or presence of varying dilutions of blank liposomes, liposomes encapsulating $400 \mathrm{ng} / \mathrm{ml}$ calcitriol and $40 \mu \mathrm{g} / \mathrm{ml} \mathrm{OVA}_{323-339}$, or equivalent final concentrations of free calcitriol. TNF, IL-6, IL-10, and IFN- $\alpha$ concentrations were measured in supernatants. Representative of 2 experiments. ${ }^{*} P<0.05,{ }^{* *} P<0.01,{ }^{* *} P<0.001$, ANOVA at highest calcitriol concentration. The percentage of dead cells was $<0.3 \%$ for all conditions at highest final calcitriol concentration. (B) Monocyte-derived DCs generated from 3 healthy human PB donors were incubated without or with $0.05 \%$ ( $5 \mathrm{nM}$ calcitriol final concentration) v/v DiD-labeled liposomes for 2 hours and washed, and then DiD fluorescence was analyzed by flow cytometry. (C) Liposome-treated DCs were incubated with $100 \mathrm{ng} / \mathrm{ml}$ lipopolysaccharide for 48 hours and then stained with HLA-DR, CD40, CD80, and CD86. ${ }^{*} P<0.05$, ANOVA. (D) Liposome-treated DCs were incubated with allogeneic CD4+ $T$ cells (ratio 1:10) for 7 days. The percentage of CD4 $4^{+} F o x p 3^{+}$allogeneic $T$ cells identified by flow cytometry is plotted for each donor pair. ${ }^{*} P<0.05$, paired $t$ test.

of endogenous antigen-specific $\mathrm{CD} 4^{+} \mathrm{T}$ cells to liposome treatment and rhPG immunization by staining splenocytes with $\mathrm{IA}^{\mathrm{d}}$-aggrecan ${ }_{89-103}$ tetramers at day 56 . Aggrecan ${ }_{89-103}$-specific $\mathrm{CD} 4^{+} \mathrm{T}$ cells expanded in diseased mice relative to adjuvant-treated control mice. In mice treated s.c. with aggrecan ${ }_{89-103} /$ calcitriol liposomes, the proportion of aggrecan-specific $\mathrm{CD} 4^{+} \mathrm{T}$ cells decreased along with tetramer staining intensity, suggesting deletion of high-affinity T cells. Furthermore, the differentiation of aggrecan ${ }_{89-103}$-specific memory populations $\mathrm{CD} 44^{\text {hi }} \mathrm{CD} 6 \mathrm{~L}^{\text {10 }} \mathrm{Tem}$ cells and CXCR5 ${ }^{+} \mathrm{PD} 1^{+} \mathrm{T}$ follicular helper (Tfh) cells - decreased, and the proportion of aggrecan ${ }_{89-103}$-specific naive $\mathrm{T}$ cells increased, while Tregs were unchanged relative to untreated diseased mice (Figure 7A). Disease severity score was negatively correlated with the proportion of aggrecan ${ }_{89-103}$-specific $^{-1}$ naive $\mathrm{T}$ cells (Figure 7A).These effects were antigen specific, as treatment with $\mathrm{OVA}_{323-339}$ /calcitriol liposomes had no effect on $\mathrm{CD} 4^{+}$aggrecan $_{89-103}$-specific $\mathrm{T}$ cells, tetramer intensity, or naive cells. Furthermore, while Treg frequency did not change, fewer Foxp3- $\mathrm{T}$ cells expressed PD1 in response to aggrecan ${ }_{89-103} /{ }$ calcitriol liposomes (Figure 7B). Together, these data demonstrate prolonged effects of liposomes on antigen-specific $\mathrm{T}$ cells. Despite repeated immunization, deleted high-affinity autoreactive differentiated memory $\mathrm{T}$ cells failed to reexpand and anergy of the remaining antigen-specific $\mathrm{T}$ cells to further antigen exposure persisted.

To assess antigen-specific tolerance after onset of inflammatory arthritis, mice were administered s.c. liposomes every 4 days from days 34 to 50 . Disease severity decreased significantly in an antigen-specific manner in mice receiving aggrecan ${ }_{89-103} /$ calcitriol liposomes but not aggrecan peptide or $\mathrm{OVA}_{323-339} /$ calcitriol $^{2}$ liposomes (Figure 7C), even though arthritis is predominantly driven by autoantibody and complement-driven mechanisms in established disease (47). In a group of mice treated with aggrecan ${ }_{89-103} /$ calcitriol liposomes 
A $\rightarrow$ Adjuvant only

- No treatment

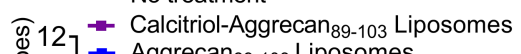

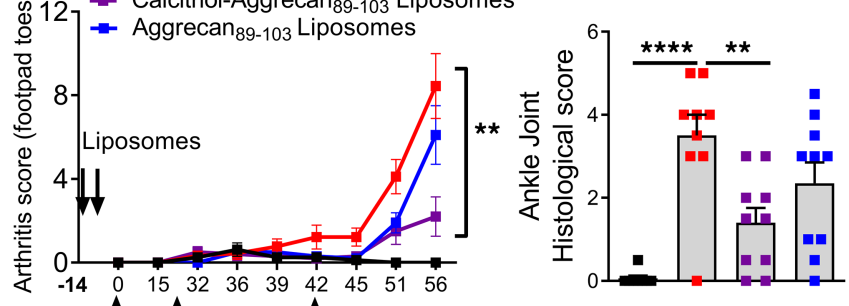

rhPG+DDA 4 Time (days) 4
Serum

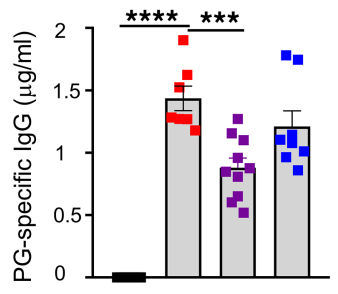

Spleen

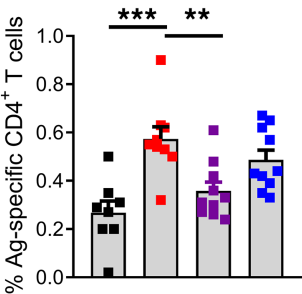

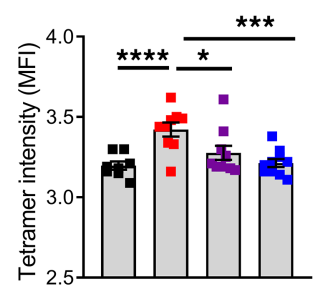
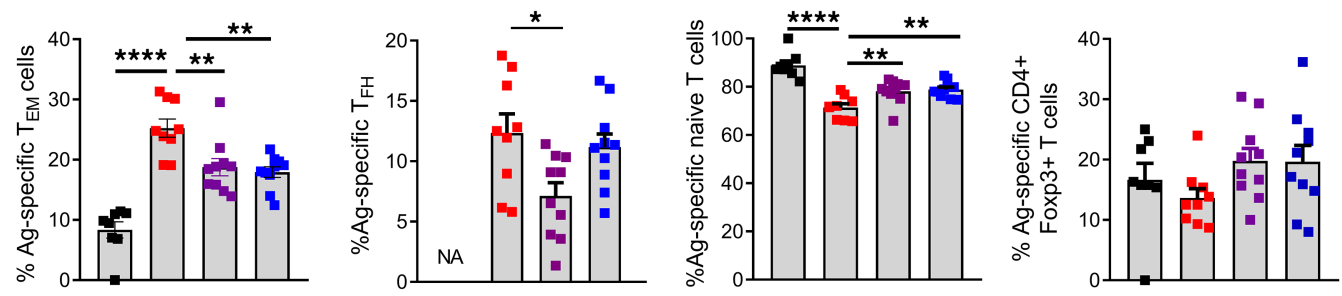

B

- Adjuvant only

- Disease, no treatment

- Calctriol/Aggrecan Liposomes

क Calcitriol/OVA Liposomes
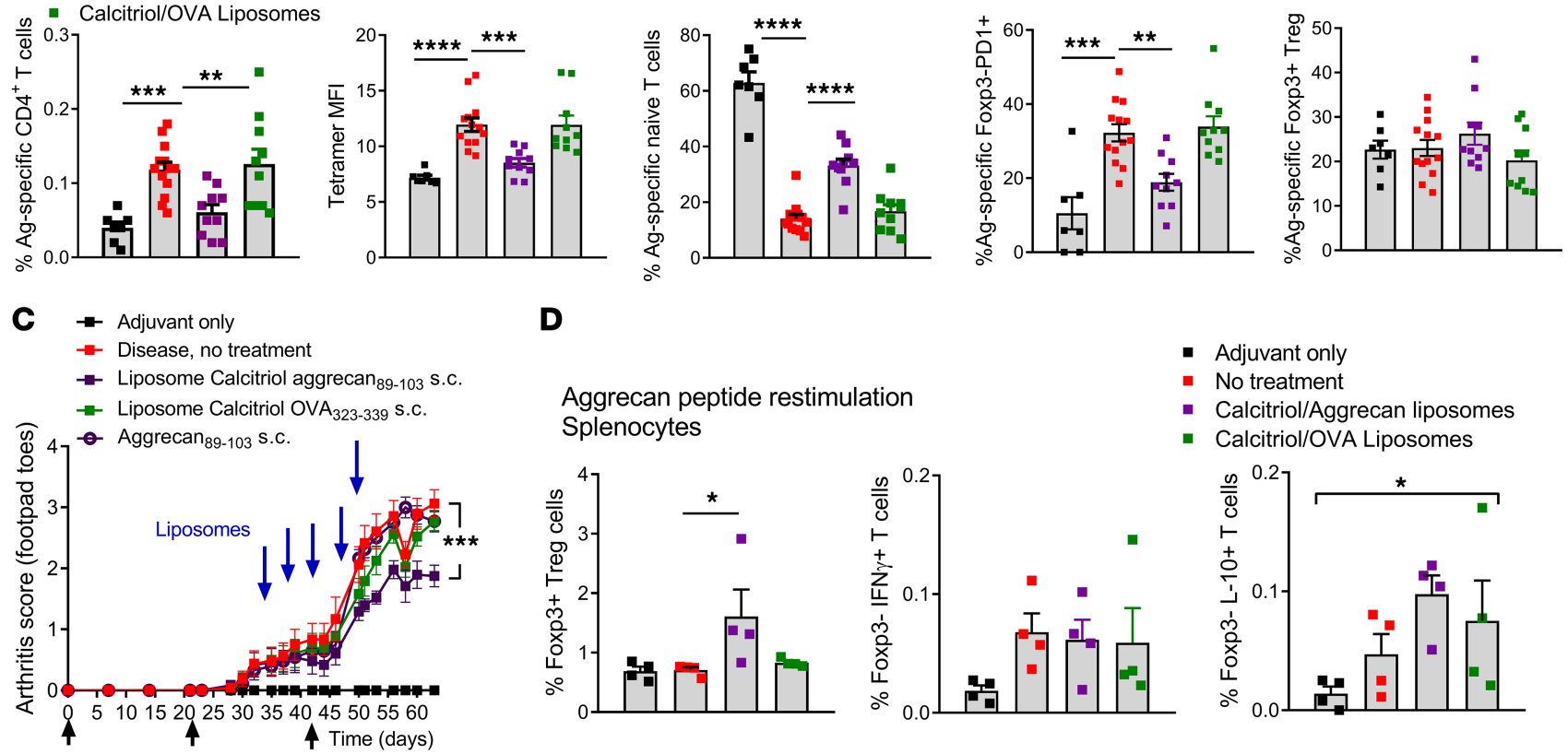

D

Aggrecan peptide restimulation Splenocytes

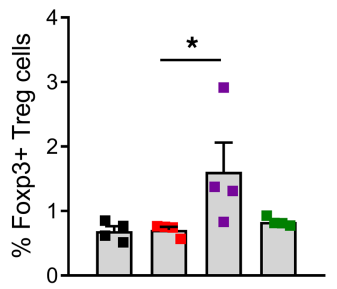

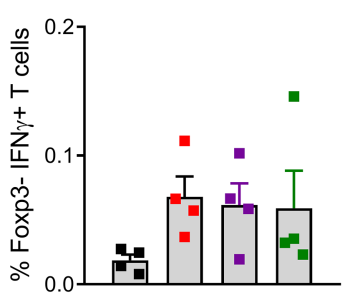

- Adjuvant only

- No treatment

- Calcitriol/Aggrecan liposomes

- Calcitriol/OVA Liposomes

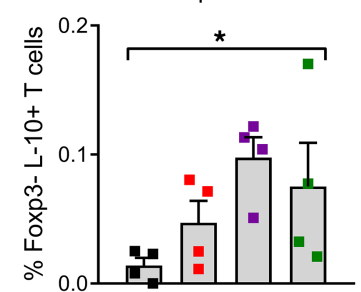

Figure 7. Liposomes encapsulating calcitriol and autoantigenic epitopes suppress autoimmune arthritis and reduce antigen-specific CD4 ${ }^{+} \mathrm{T}$ cells. (A) For induction of PGIA, BALB/CAnNCrl mice were primed i.p. with rhG1-PG fusion protein and DDA adjuvant and then boosted on days 21 and 42 . Mice injected with DDA were used as disease negative controls. Mice were administered $100 \mu$ l saline or liposomes as shown s.c. into the tail base on days -14 and -7 . Arthritis severity was scored visually based on swelling and redness of the front and hind paws weekly until day 21 and then twice weekly until the endpoint on day 55 ( $n=12$ /group, DDA alone 8/group). Histological scores were evaluated from H\&E-stained slides, and PG-specific IgG1 antibodies were quantified by ELISA in sera at the endpoint. Correlation of disease score and aggrecan-specific naive T cells. $R^{2}=0.3, P<0.0001$. IA $A^{d}$-aggrecan ${ }_{89-103}$ tetram-

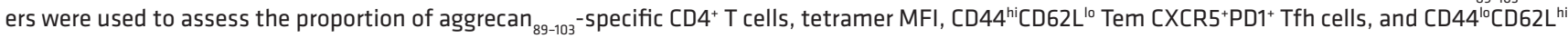


naive and Foxp3 $3^{+}$cells in spleen. (B) Experimental conditions as in $\mathbf{A}$, with liposomes administered as shown and analysis of proportion of tetramer T cells, tetramer MFI, naive and Foxp3-PD1+ T cells, and Foxp3 ${ }^{+}$Tregs ( $n=12$ /group, DDA alone $8 /$ group). (C) PGIA was induced as in A, and mice were administered $100 \mu \mathrm{L}$ saline or liposomes as shown s.c. into the tail base on days 32, 36, 40, 44, and 48 ( $n=12 /$ group, DDA alone $8 /$ group). (D) Experimental conditions as in C, in which splenocytes from an additional 4 mice in liposome-treated and control groups were restimulated for 7 days with aggrecan ${ }_{89-103}$ and then analysis of Foxp3 ${ }^{+}$Tregs and Foxp3-IFN- $\gamma$ and IL-10+ T cells by flow cytometry. ${ }^{*} P<0.05 ;{ }^{*} P<0.01 ;{ }^{* *} P<0.001 ;{ }^{* * * *} P<0.0001$, ANOVA with Tukey's multiple comparison test; disease scores compared at endpoint.

that was analyzed on day 42, Foxp3 ${ }^{+}$Tregs and Foxp3-IL- $10^{+} \mathrm{T}$ cells expanded upon restimulation with

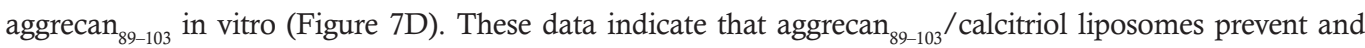
suppress existing arthritis in an antigen-specific manner and provide evidence that both Foxp $3^{+}$and Tr1-type regulatory responses are generated after antigen reexposure in vitro.

To determine whether peptide/calcitriol encapsulated liposomes have more generalized potential in treating inflammatory autoimmune disease, we studied Goodpasture's disease, a severe inflammatory vasculitis affecting the kidneys and the lungs. This disease is directed by autoreactive HLA-DR15-restricted collagen IV $\alpha 3_{135-145}$-specific CD4 ${ }^{+} \mathrm{T}$ cells and can be modeled by immunizing HLA-DR15-transgenic $F_{c g r} 2 b^{-1-}$ mice with $\alpha 3_{135-145}(48,49)$. In mice treated twice with liposomes encapsulating calcitriol and $\alpha 3_{135-145}$ peptide, the development of glomerulonephritis and albuminuria was reduced in an antigen-specific manner. Reduced injury was associated with fewer intrarenal $\alpha 3_{135-145}$-specific CD $4^{+}$Foxp $3^{-}$Teffs. The proportion of $\alpha 3_{135-145}$-specific Tregs increased in the spleen (Figure 8, A and B). When treatment was delayed until after autoimmunity was established (day 21), histological injury also was limited and was associated with a higher proportion of splenic $\alpha 3_{135-145}$-specific Foxp $3^{+}$cells (Figure 8C). To assess the immune response to liposomes encapsulating calcitriol and $\alpha 3_{135-145}$ or $\mathrm{OVA}_{323-339}$ in detail, we administered liposomes twice and then immunized mice with $\alpha 3_{135-145}$, and assessed $\alpha 3_{135-145}$-specific CD $4^{+} \mathrm{T}$ cells in pooled spleen and dLNs using HLA-DR15-transgenic Fc $\gamma$ RIIb intact mice. The proportion of antigen-specific CD44 ${ }^{\text {hi }} \mathrm{CD} 62 \mathrm{~L}^{\text {lo }}$ Tem cells and CXCR5 ${ }^{+} \mathrm{PD} 1^{+} \mathrm{Tfh}$ cells decreased, naive and $\mathrm{CD} 73^{+}$memory $\mathrm{T}$ cells increased, and IL-17A and IFN- $\gamma$ production in response to $\alpha 3_{135-145}$ decreased in mice treated with calcitriol/ $\alpha 3_{135-145}$ liposomes (Figure 8D). Thus, differentiation of autoantigen-specific memory CD4 ${ }^{+} \mathrm{T}$ cells, vasculitis severity, and proinflammatory cytokines are suppressed by calcitriol/ $\alpha 3_{135-145}$ liposomes in experimental Goodpasture's disease.

\section{Discussion}

Autoantigen-specific immunological tolerance is the preferred strategy for future control of autoimmune diseases. However, induction of tolerance may be challenging when inflammatory signals drive activation of DCs from their resting state, resulting in the promotion of autoreactive memory $\mathrm{T}$ cell expansion and differentiation. Genomic and phenotypic evidence strongly implicates Tem cells, Tfh cells, and Tregs in the pathogenesis of RA and Goodpasture's disease (49-51). Furthermore, high-risk HLA alleles, autoantibodies, inflammatory biomarkers, and $\mathrm{CD}^{+}$tetramers may identify patients with RA and small-vessel vasculitides who may be suitable for antigen-specific immunotherapeutic interventions (52-55). Therefore, preclinical development of antigen-specific strategies that control autoreactive memory $\mathrm{T}$ cells under inflammatory conditions is required in a clinically tractable format. We show here that $2-5$ administrations of peptide/ calcitriol liposomes suppressed expansion, differentiation, and function of memory $\mathrm{T}$ cells and suppressed the severity of inflammatory arthritis and humanized Goodpasture's vasculitis models before and after onset in an antigen-specific and calcitriol-dependent manner. $\mathrm{CD} 4^{+}$antigen-specific Teffs could be identified from the polyclonal repertoire with pMHC tetramers. Thus, pMHC-II multimers have excellent potential as companion biomarkers for clinical studies of antigen-specific immunotherapy using peptide/calcitriol liposomes to induce antigen-specific $\mathrm{CD} 4^{+} \mathrm{T}$ cell tolerance in vivo. Consistent with models of transgenic antigen, after s.c. administration of liposomes antigen-specific T cells appeared to be controlled by probable deletion of high-affinity memory $\mathrm{T}$ cells and prolonged unresponsiveness of residual antigen-specific T cells. Furthermore, ex vivo antigen exposure potentiated Treg expansion. Our data demonstrate that peptide/calcitriol liposomes promote multiple mechanisms of peripheral tolerance toward widely expressed autoantigens.

DCs upregulate capacity for antigen presentation, including MHC and costimulatory molecules and cytokines in response to adjuvant-induced stimulation of $\mathrm{NF}-\kappa \mathrm{B}$, reinforced by cognate interactions with responding $\mathrm{T}$ cells $(10,56)$. Nanoparticles encapsulating peptide and immunomodulators, such as rapamycin, curcumin, or an aryl hydrocarbon receptor ligand, were shown to induce antigen-specific tolerance in animal models (13-16). However, the mechanism of tolerogenic signal transmission from DCs 
- Saline

A - Calcitriol OVA $323-339$ liposomes

- Calcitriol $\alpha 3_{135-145}$ liposomes
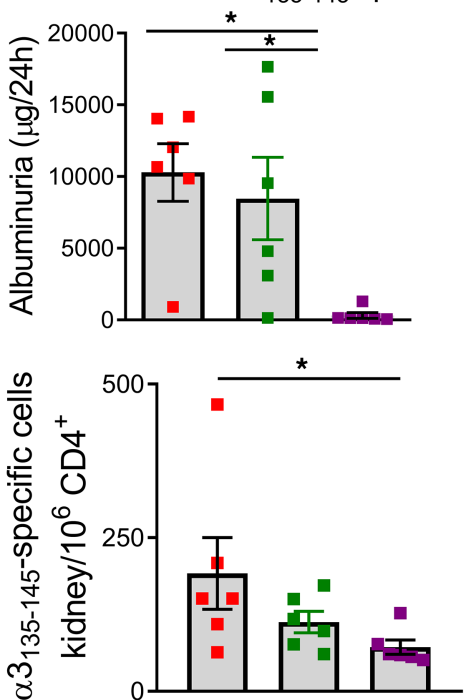

C

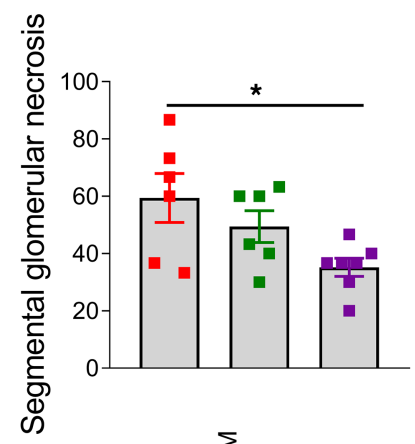

D
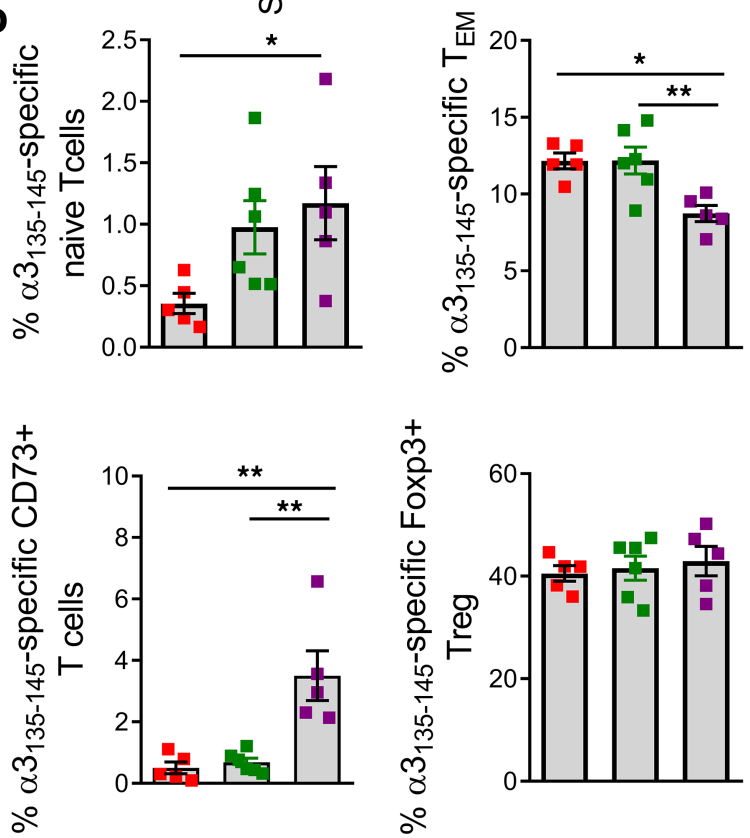
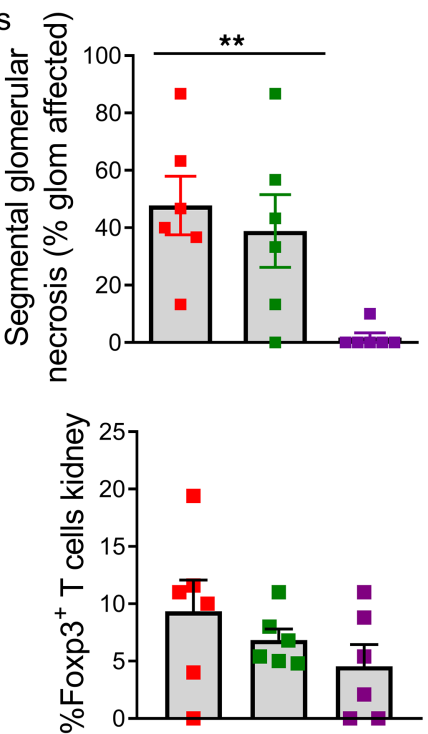
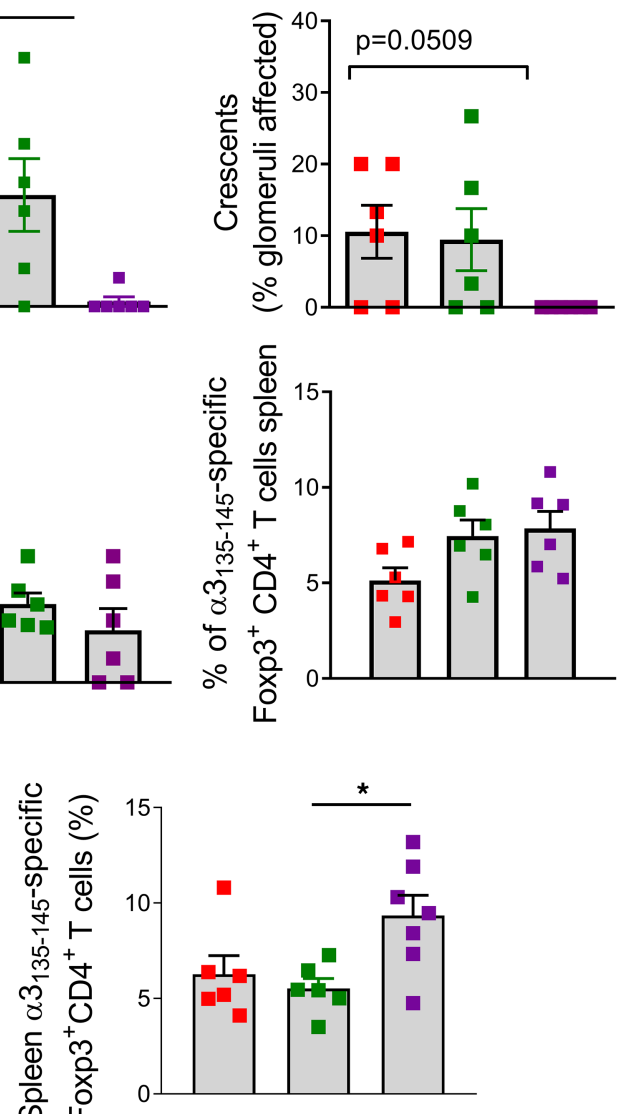

B
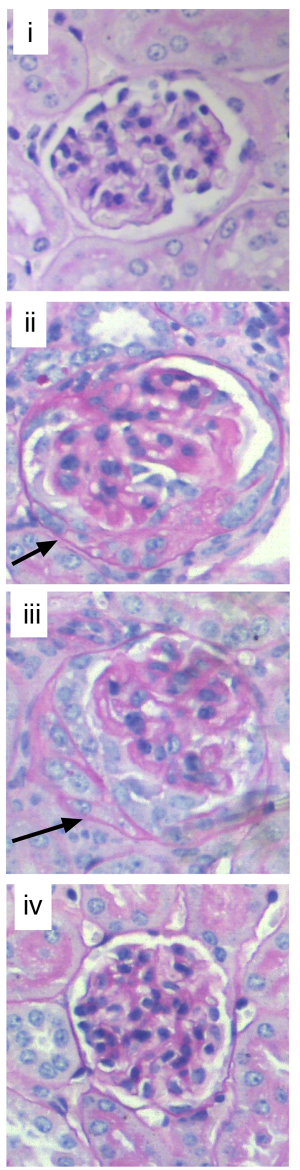
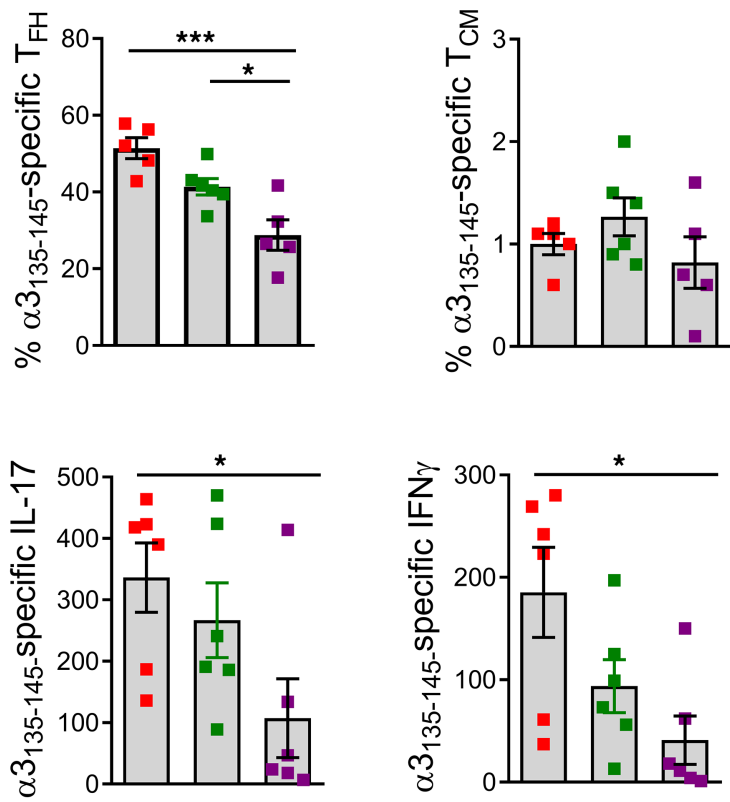

Figure 8. Liposomes encapsulating calcitriol and autoantigenic epitopes suppress autoimmune Goodpasture's vasculitis and reduce antigenspecific CD4 ${ }^{+}$T cells. (A) HLA-DR15-transgenic, mouse MHC class II ${ }^{-/-} \mathrm{Fcgr2b}^{-1-}$ mice were primed s.c. with $\alpha 3_{135-145}$ in CFA and boosted twice with $\alpha 3_{135-145}$ in IFA. Mice were administered saline, calcitriol/OVA $A_{323-339}$ liposomes, or calcitriol/ $\alpha 3_{135-145}$ liposomes s.c. into the tail base on days -14 and -7 ( $n=6$ /group). At day 42 , functional renal injury was measured by albuminuria, and glomerular injury was assessed histologically for segmental glomerular necrosis and crescents. Teffs in kidney were enumerated, and DR15- $\alpha 3_{135-145}$ tetramers were used to assess the number of $\alpha 3_{135-145}-$ specific intrarenal Foxp3- cells and Foxp3 $3^{+} \mathrm{CD}^{+} \mathrm{T}$ cells and the percentage of Foxp3 $3^{+}$CD4 $4^{+}$T cells in spleen. (B) Periodic acid-Schiff (PAS) staining of glomeruli 
from DR15-transgenic mice: naive, saline, calcitriol/OVA ${ }_{323-339}$ liposomes, calcitriol/ $\alpha_{135-145}$ liposomes; representative of 6 mice/group. Accumulation of PAS-positive (pink) stain denote areas of segmental glomerular necrosis, with crescent formation indicated by the accumulation of cells in Bowman's space (arrows). Original magnification, $\times 200$. (C) Mice ( $n=5-6 /$ group) were primed as in A and treated as shown on days 21 and 28 . On day 42, glomerular injury was assessed histologically for segmental glomerular necrosis and crescents, and the proportion of $\alpha 3_{135-145}-5$ pecific splenic

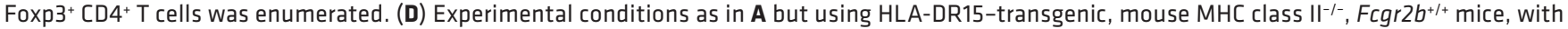

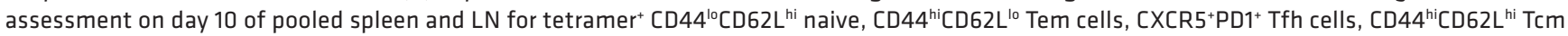
cells, CD73+ Tem cells, and Foxp3 $3^{+}$Tregs. IL-17A and IFN- $\gamma$ production in ILN by ELISPOT after stimulation with $\alpha 3_{135-145^{\circ}}{ }^{*} P<0.05 ;{ }^{* *} P<0.01 ;{ }^{* * *} P<$ 0.001 (ANOVA with Tukey's or Dunnett's multiple comparison test).

taking up such nanoparticles to responding antigen-specific $\mathrm{T}$ cells is unclear. We show that in a primed setting CD11 b ${ }^{+}$Ly6C ${ }^{-}$DCs taking up liposomes express high levels of PD-L1 and that OVA/calcitriol liposomal targeting increases their frequency and suppresses their MHC class II expression in dLNs. This PD-L1 is required for control of T cell expansion and induction of Foxp $3^{+}$and IL-10 $10^{+}$Tregs. These data challenge the notions that tolerance induction requires antigen presentation by "resting" DCs or quiescent disease to take advantage of low levels of costimulatory molecule expression (57). Rather, T cell-mediated DC cross-talk underpins DC migration to dLNs and regulation of antigen presentation. Furthermore, we show that Treg induction in mice and humans, disease suppression, and reduced CD11 b ${ }^{+}$Ly6C ${ }^{-}$DC pMHC density are calcitriol dependent. This calcitriol-dependent mechanism is consistent with evidence that reduced pMHC increases Treg frequency and T cell PD1 expression (58, 59). Sustained PD1 is a feature of T cell anergy or exhaustion in response to chronic antigen exposure in cancer, infection, and various tolerance settings, including $\mathrm{T}$ cell regulation in mesenteric LN (60-63). Sustained PD1 expression was also shown to suppress Tfh cell development (64), consistent with the effects of peptide/calcitriol liposomes observed in this study.

Antigen-specific tolerance occurred within days after a single liposome administration, which increased after 2 doses and required both antigen and calcitriol encapsulation in order to achieve sufficient antigen delivery for antigen-specific T cell PD1 induction. Thus, liposome codelivery of peptide with calcitriol in the lipid and aqueous phase, respectively, permits precise antigen dosing to and modulation of targeted dLN DCs, avoiding the multi-dose escalation required to achieve unresponsiveness with peptide alone (65). In conclusion, peptide/calcitriol liposomes leverage PD-L1 expression for antigen-specific regulation via T cell PD1 and represent a targeted immunotherapeutic strategy for treatment of systemic inflammatory autoimmune diseases.

\section{Methods}

Mice. BALB/c mice were purchased from the Animal Resources Centre (BALBcArc) and Charles River (BALB/cAnNCrl). For Goodpasture's disease, HLA-DR15-transgenic mice were HLA-DRA1*01:01/ $H L A-D R B 1^{*} 15: 01$-transgenic, mouse MHC class $\mathrm{II}^{-/-}$and $\mathrm{Fcgr}_{2 b^{-/-}}$and for day 10 immune response experiments, mice were HLA-DRA1*01:01/HLA-DRB1*15:01-transgenic, mouse MHC class $\mathrm{II}^{-/-}$and Fcgr2b intact, mice were Fc $\gamma$ RIIb intact. HLA-DR15-transgenic mice were on a mixed C57BL/6, C57BL/10, and DBA/2 background (48). Mice were sex matched within experiments and used at between 8 and 20 weeks of age. All mice were kept under specific pathogen-free conditions either at the Biological Research Facilities of Translational Research Institute or Monash Medical Centre.

Liposome preparation. Liposomes were prepared either by thin-film hydration or microfluidic technique. Briefly, EPC (Avanti Lipids) and EPG (Avanti Lipids) were dissolved in a 9:1 ratio in chloroform/ethanol and evaporated to prepare a thin film under reduced pressure in a rotary evaporator. The film was hydrated with HEPES buffer ( $\mathrm{pH}$ 7.4), and the resulting liposomes were subjected to 5 freeze-thaw cycles followed by sonication using a 3-mm probe at $40 \%$ amplitude (Sonics and Materials). For the preparation of drug-loaded liposomes, $400 \mathrm{ng} / \mathrm{mL}$ calcitriol (Dishmann) was added to the lipid film while $30 \mu \mathrm{g} / \mathrm{mL}$ peptide (Ovalbumin $_{323-339}$ (ISQAVHAAHAEINEAGR), Aggrecan ${ }_{89-103}$ (ATEGRVRVNSAYQDK), and $\alpha 3(I V) N C 1_{136-146}$ (GWISLWKGFSF), and $\mathrm{HA}_{126-138}$ (HNTNGVTAASSHE, Auspep Mimotope) was added to the aqueous phase during hydration. Microfluidic liposomes were formulated with the benchtop NanoAssemblr instrument (Precision Nano-Systems Inc.). Two inlet streams comprised EPC and EPG dissolved in methanol and HEPES buffer, and flow rates and flow ratios between the 2 inlet streams were controlled with syringe pumps. Nonentrapped material was separated by dialysis and ultrafiltration. Liposomes were injected into mice in $100 \mu \mathrm{L}$ volume s.c. (at base of tail, flank, or neck scruff) unless otherwise specified.

Liposome biodistribution. For in vivo uptake studies, BALB/c female mice (6-8 weeks) were primed with OVA/QuilA, 24 hours before injection with unlabeled liposomes, single color-labeled liposomes constructed 
with BODIPY FL-labeled calcitriol (66), BODIPY TXR-labeled 1,2-Dihexadecanoyl-sn-glycerol-3-phosphoethanolamine triethylammonium salt (DHPE, Molecular Probes), or dual-labeled liposomes (constructed with FL-calcitriol and TXR-DHPE). After 15 minutes and 5 hours the animals were euthanized and dLNs were harvested for single cell uptake assessment by flow cytometry. In some experiments, whole dLNs were harvested and embedded in OCT and snap frozen. Cryosections $(10 \mu \mathrm{m})$ were fixed in ice-cold acetone (MilliporeSigma) for 10 minutes and dried at room temperature. Sections were stained at room temperature with anti-IA/IE, anti-CD11c. Slides were imaged using an Olympus Confocal Laser Scanning Microscope with an $\times 20$ objective and acquiring a tiled image. For in vitro uptake, BODIPY FL-labeled calcitriol liposomes and BODIPY TXR-labeled DHPE liposomes were cocultured with RAW 264.7 macrophages (ATCC) for 2 hours before assessment of uptake by confocal microscopy.

Radiolabeled biodistribution studies were conducted on 10-12 week female Swiss outbred mice. Briefly, liposomes were prepared by incorporating ${ }^{3} \mathrm{H}$-calcitriol and ${ }^{14} \mathrm{C}$-dipalmitoyl phosphatidylcholine, and $100 \mu 1$ was administered via s.c. injection. Animals were euthanized at predetermined time intervals ( 5 minutes, 15 minutes, 30 minutes, 1 hour, 2 hours, and 24 hours), and plasma and tissue samples (liver, spleen, kidneys, lungs, brain, dLNs, and tissue from site of injection) were harvested and stored at $-80^{\circ} \mathrm{C}$ until further analysis by digestion of the tissues and scintillation counting as described previously (67).

For remaining uptake and distribution experiments, liposomes were stained with lipophilic membrane dyes (DiI or DiR, Molecular Probes) at a final concentration of $25 \mu \mathrm{g} / \mathrm{mL}$ dye for at least 30 minutes at $4^{\circ} \mathrm{C}$. $\mathrm{BALB} / \mathrm{c}$ female mice were left untreated or primed with OVA/QuilA for 24 hours before injection with 100 $\mu \mathrm{L}$ DiR-labeled OVA ${ }_{323-339}$ liposomes s.c. at the tail base. After 24 hours, the IVIS Spectrum In Vivo Imaging System (Perkin Elmer) was used to assess in vivo distribution of fluorescently labeled liposomes first in wholebody images (imaging parameters: excitation: $710 \mathrm{~nm}$,emission: $760 \mathrm{~nm}$, binning: 4, exposure: 5 min and aperture: f1) and subsequently in isolated organs and LNs with average radiant efficiency $\left[\mathrm{p} / \mathrm{s} / \mathrm{cm}^{2} / \mathrm{steradian}\right] /$ $\left[\mu \mathrm{W} / \mathrm{cm}^{2}\right]$ calculated for each individual organelle. In other experiments, single cellular DiI liposome uptake was assessed by flow cytometry as described below.

Adoptive cell transfers and in vivo blocking. Naive DO11.10 cells for transfer were collected from spleens and pooled LNs (inguinal, axillary, brachial, mesenteric) and crushed through Falcon $70 \mu \mathrm{m}$ cell strainer (Corning, BD Biosciences). Erythrocytes in splenocyte suspensions were lysed in ACK buffer. Cells were prepared in PBS, labeled when required with CFSE or CTV (Molecular Probes), and $5 \times 10^{6}$ cells were injected into mice i.v.

For in vitro generation of OVA-specific $\mathrm{CD} 4^{+}$memory $\mathrm{T}$ cells, spleens, and pooled LNs isolated from DO11 mice were cultured as described previously (57), except 10\% FBS was substituted for mouse serum, resulting in a mixed population of effector memory and central memory $\mathrm{T}$ cells, as assessed by CD62L and CD44 expression (Supplemental Figure 6). OVA/QuilA (100 $\mu \mathrm{g}$ OVA, MilliporeSigma; $20 \mu \mathrm{g}$ QuilA, Accurate Chemical) was used for OVA priming. For in vivo blocking of PD-L1, $200 \mu \mathrm{g}$ anti-PD-L1 antibody (clone 10F.9G2, BioXCell) was injected i.p. every 3 days for duration of experiment. Control mice received same amount isotype anti-KLH antibody (clone LTF-2, BioXCell).

For in vitro Treg assay, dLN CD $45.2^{+} \mathrm{CD} 19^{-} \mathrm{MHCII}^{+} \mathrm{CD} 11 \mathrm{c}^{+} \mathrm{DCs}, \mathrm{CD} 45.2^{+} \mathrm{CD} 19^{+} \mathrm{MHCII}^{+} \mathrm{B}$ cells, and CD45.2- stromal cells were isolated from mice administered OVA/QuilA and liposomes 24 hours previously. APCs were cultured with DO11.10 CD4 ${ }^{+} \mathrm{T}$ cells in a ratio of $1: 2$ in the presence of $50 \mathrm{ng} / \mathrm{ml}$ IL-2. CD $25^{+} \mathrm{Foxp}^{+} \mathrm{T}$ cells were enumerated by FACS 5 days later. Positive control DO11.10 CD4 ${ }^{+} \mathrm{T}$ cells were cultured with $2 \mu \mathrm{g} / \mathrm{ml}$ anti-CD3, $0.5 \mu \mathrm{g} / \mathrm{ml}$ anti-CD28, $5 \mathrm{ng} / \mathrm{ml} \mathrm{rhTFG}-\beta, 50 \mathrm{ng} / \mathrm{ml} \mathrm{IL-2,1} \mu \mathrm{g} / \mathrm{ml}$ anti-IL-4, and $1 \mu \mathrm{g} / \mathrm{ml}$ anti-IFN- $\gamma$.

Proteoglycan-induced arthritis. CHO-K1 (Chinese hamster ovary) cells transfected with recombinant human aggrecan 1 proteoglycan-Xa-mFc2a (rhG1-PG-Xa-mFc2a) fusion protein were a gift from Tibor Glant, Rush University, Medical Centre, Chicago, Illinois, USA. Purified rhG1-PG-Xa-mFc2a fusion protein was isolated from cell culture media at the National Biologics Facility, Queensland node, AIBN, The University of Queensland. For PGIA induction, $80 \mu \mathrm{g}$ rhG1-PG-Xa-mFc2a fusion protein was emulsified with $2 \mathrm{mg}$ dimethyldioctadecyl-ammonium bromide (DDA; MilliporeSigma) adjuvant, and $300 \mu \mathrm{l}$ was injected i.p. to BALB/cAnNCrl mice on days 0, 21, and 42 as described previously (68). Mice injected with DDA were used as disease negative controls. Arthritis severity was scored visually based on the degree of swelling and redness of the front and hind paws (score range from 0 to 16), weekly until day 21, and then twice weekly until the endpoint on day 55 (69). H\&E-stained paraffin-embedded ankle joint sections were scored as previously described (70). Mice were randomly assigned to treatment and control groups, and all clinical and histological measurements were performed by independent researchers in a blinded manner. 
Experimental autoimmune Goodpasture's disease. Disease was induced in HLA-DRB1*15:01-transgenic, mouse MHC class $\mathrm{II}^{-/-} \mathrm{Fcgr}_{\mathrm{c}} \mathrm{b}^{-/-}$mice by subcutaneous injections of $100 \mu \mathrm{g} \alpha 3_{135-145}$ emulsified initially in Freund's complete adjuvant and then subsequently in Freund's incomplete adjuvant on days 0, 7, and 14; mice were culled on day 42 . Mice were administered $100 \mu \mathrm{L}$ s.c. saline, calcitriol/OVA ${ }_{323-339}$ liposomes, or calcitriol/ $\alpha 3_{135-145}$ liposomes to the tail base on days -14 and -7 or days 21 and 28 . Albuminuria as a measure of functional renal injury was determined by 24-hour urinary albumin, quantified by ELISA (Bethyl Laboratories). Histological injury was assessed by scoring glomerular necrosis and crescents on formalin-fixed paraffin-embedded kidney sections stained with periodic acid-Schiff.

Cell preparation, staining, and flow cytometry. For flow cytometric analysis, spleens and LNs were manually disrupted by pressing through strainers, erythrocytes were lysed in ACK lysis buffer, and cells were washed in PBS/0.1\%BSA/EDTA. Kidneys were digested with a solution containing $0.125 \%(\mathrm{w} / \mathrm{v})$ collagenase D (Roche) and $0.1 \%$ (w/v) DNase I (Roche) and enriched for $\mathrm{CD} 45^{+}$cells by MACS using mouse CD 45 microbeads (Miltenyi Biotec). The following fluorochrome-conjugated antibodies were used for flow cytometry: antimouse CD3 (clone 144-2C11), anti-mouse CD4 (clone GK1.5), anti-mouse/human CD11b (clone M1/70), anti-mouse CD11c (clone N418), anti-mouse FoxP3 (clone FJK-16s), anti-mouse CD39 (clone Duha59), antimouse CD73 (clone TY/11.8), anti-mouse CD103 (clone 2E7), anti-mouse TCR DO11.10 (clone KJ1-26), anti-mouse CD19 (clone 6D5), anti-mouse MHC class II I-A/I-E (clone M5/114.15.2), anti-mouse CXCR5 (clone L138D7), anti-mouse PD1 (clone 29F.1A12), anti-mouse CD44 (clone IM7), anti-mouse CD62L (clone MEL-14), anti-human/mouse ICOS (clone C398.4A), anti-mouse CD45.2 (clone 104), B220 (clone RA3-6B2), F4/80 (clone BM8), PD-L1 (clone 10F.9G2), Siglec-H (clone 551), Ly6C (clone HK1.4), Ly6G (clone 1A8), CD8 $\alpha$ (clone 53-6.7) CD40 (clone 3/23), CD80 (clone 16-10A1), CD86 (clone GL-1), IFN- $\gamma$ (clone XMG1.2), and IL-10 (clone JES5-16E3) were obtained from Biolegend. Anti-mouse FoxP3 (clone FJK-16s) was sourced from eBioscience. Lineage dump mix for myeloid experiments included anti-CD3 and anti-CD19, and lineage dump mix for tetramer analysis included anti-CD19 and anti-CD11c. Kidney cells were incubated with anti-mouse CD16/CD32 (BD Biosciences) to block Fc-binding sites. Intracellular staining for Foxp3 and cytokine staining for IFN- $\gamma$ and IL-10 used the Foxp3/Transcription Factor Staining Buffer Set (eBioscience) or Fixation and Perm/Wash buffers (Biolegend). For cytokine staining, cells were restimulated in vitro with $10 \mu \mathrm{g} / \mathrm{mL}$ peptide for 24 hours, and Brefeldin A added for the final $3-4$ hours. Samples

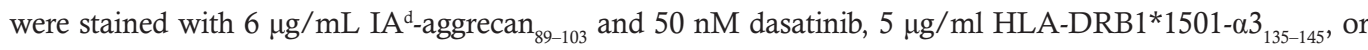
remained unstained for 1 hour in the dark. Samples stained with HLA-DRB1*1501- $\alpha 3_{135-145}$ were enriched for tetramer-positive cells using anti-PE microbeads (Miltenyi Biotec). Cells were then stained with Live/Dead Aqua or Near-IR (Molecular Probes), lineage dump mix, and the specific mAb cocktail. Dead cells were excluded with Live/Dead staining, doublets were excluded on morphology, and gates were set based on FMO and isotype controls. Bead-based cell counts used Flow Count Fluorospheres (BD).

For generation of human moDCs, CD14 $4^{+}$monocytes isolated from PBMCs of healthy human donors were cultured in complete medium in the presence of $800 \mathrm{U} / \mathrm{ml} \mathrm{rhGM}-\mathrm{CSF}$ and $400 \mathrm{U} / \mathrm{ml} \mathrm{rhIL}-4$ (Peprotech) for 6 days. After incubation with $0.05 \%$ v/v DiD-labeled calcitriol/OVA ${ }_{323-339}$ liposomes for 2 hours, DiD fluorescence was analyzed by flow cytometry. For phenotypic analysis, moDCs were incubated with $0.05 \%(5 \mathrm{nM}$ calcitriol final concentration) calcitriol/OVA ${ }_{323-339}$ or blank liposomes for 2 hours followed by either $100 \mathrm{ng} /$ $\mathrm{ml}$ lipopolysaccharide for 48 hours or allogeneic $\mathrm{CD} 4^{+} \mathrm{T}$ cells (ratio 1:10) for 7 days. LPS-treated DCs were stained with HLA-DR, CD40, CD80, and CD86. T cells were stained with CD3, CD4, CD25, and Foxp3. We acquired samples on a Gallios or Fortessa $\times 20$ flow cytometer and used Kaluza software v1.3 for analysis (Beckman Coulter). For analysis of cytokine production, PBMCs were stimulated for 18 hours with $10 \mu \mathrm{g} / \mathrm{ml}$ $\mathrm{CpG}$ in the absence or presence of varying dilutions of blank liposomes, liposomes encapsulating $400 \mathrm{ng} / \mathrm{ml}$ calcitriol and $40 \mu \mathrm{g} / \mathrm{ml} \mathrm{OVA}_{323-339}$, or equivalent final concentrations of free calcitriol. Secreted TNF, IL-6, and IL-10 levels were measured by cytokine bead array (BD) in supernatants and IFN- $\alpha$ by ELISA.

ELISPOT. For IFN- $\gamma$ and IL-17A ELISPOTs, LN samples were plated in duplicate and incubated overnight with $\alpha_{135-145}$ or RPMI complete medium.

Statistics. Graphs were generated using GraphPad Prism 7 (GraphPad Software). All data are presented as mean \pm SEM. Normally distributed data were analyzed by 2-tailed $t$ test or 1-way ANOVA as appropriate, with Sidak's or Tukey's multiple comparisons correction, and nonnormally distributed data were analyzed by Mann-Whitney and Kruskal Wallis tests. Time-course or multiparameter data were analyzed by 2-way ANOVA with Tukey's or Dunnett's multiple comparisons post hoc test. $P$ values of less than 0.05 were considered significant. 
Study approvals. Animal procedures and experiments were approved by The University of Queensland and Monash University Animal Ethics Committees. Human experiments were approved by the Metro South Health and Hospital Services and The University of Queensland Human Research Ethics Committees. Subjects provided informed consent prior to their participation in the study.

\section{Author contributions}

RT had full access to all of the data in the study and takes full responsibility for the integrity of the data and the accuracy of the data analysis. RT, BJO, RG, MT, XL, and HJN were responsible for study concept and design. RG, HJN, MT, XL, JDO, MH, SH, KJR, YTT, SC, KC, SH, BZ, SY, MB, NG, HR, JR, HHR, BJB, RM, DJS, DGB, LM, ARK, BJO, and RT acquired, analyzed, and interpreted data. RT, RG, MT, and ARK drafted the manuscript. RG, HJN, MT, XL, JDO, MH, SH, KJR, YTT, SC, KC, SH, BZ, SY, MB, NG, HR, JR, HHR, BJB, RM, DJS, DGB, LM, ARK, BJO, and RT provided critical revision of the manuscript for important intellectual content. RT obtained funding.

\section{Acknowledgments}

We thank Anna Pham, Gisela Ramirez, and Lisa Kaminskas for technical assistance with the biodistribution studies. We thank Jeff Hou and Martina Jones (National Biologicals Facility, Australian Institute for Bioengineering and Nanotechnology, The University of Queensland) for purification of rhG1-PG and Tibor Glant (Rush University) for supplying the rhG1-PG expression plasmid. This work was supported by National Health and Medical Research Council (NHMRC) grants 1071822 and 1083192. RT is supported by a NHMRC Senior Research Fellowship and Arthritis Queensland. MT is supported by a fellowship from Arthritis Australia. JR is an Australian Research Council Laureate Fellow.

Address correspondence to: Ranjeny Thomas, The University of Queensland Diamantina Institute, Translational Research Institute, 37 Kent Street, Woolloongabba, Queensland 4102, Australia. Phone: 61.7.3443.6960; Email: ranjeny.thomas@uq.edu.au.

1. Johnston SS, Turpcu A, Shi N, Fowler R, Chu BC, Alexander K. Risk of infections in rheumatoid arthritis patients switching from anti-TNF agents to rituximab, abatacept, or another anti-TNF agent, a retrospective administrative claims analysis. Semin Arthritis Rheum. 2013;43(1):39-47.

2. Yates M, et al. EULAR/ERA-EDTA recommendations for the management of ANCA-associated vasculitis. Ann Rheum Dis. 2016;75(9):1583-1594.

3. Winthrop KL, et al. The unmet need in rheumatology: Reports from the targeted therapies meeting 2017. Clin Immunol. 2018;186:87-93.

4. Banchereau J, Steinman RM. Dendritic cells and the control of immunity. Nature. 1998;392(6673):245-252.

5. Thomas R. Dendritic cells as targets or therapeutics in rheumatic autoimmune disease. Curr Opin Rheumatol. 2014;26(2):211-218.

6. O'Sullivan BJ, MacDonald KP, Pettit AR, Thomas R. RelB nuclear translocation regulates B cell MHC molecule, CD40 expression, and antigen-presenting cell function. Proc Natl Acad Sci USA. 2000;97(21):11421-11426.

7. Bell GM, et al. Autologous tolerogenic dendritic cells for rheumatoid and inflammatory arthritis. Ann Rheum Dis. 2017;76(1):227-234

8. O'Sullivan BJ, et al. Immunotherapy with costimulatory dendritic cells to control autoimmune inflammation. J Immunol. 2011;187(8):4018-4030.

9. Martin E, et al. Antigen-specific suppression of established arthritis in mice by dendritic cells deficient in NF-kappaB. Arthritis Rheum. 2007;56(7):2255-2266

10. Martin E, O'Sullivan B, Low P, Thomas R. Antigen-specific suppression of a primed immune response by dendritic cells mediated by regulatory T cells secreting interleukin-10. Immunity. 2003;18(1):155-167.

11. Li M, et al. Immune modulation and tolerance induction by RelB-silenced dendritic cells through RNA interference. J Immunol. 2007;178(9):5480-5487.

12. Benham H, et al. Citrullinated peptide dendritic cell immunotherapy in HLA risk genotype-positive rheumatoid arthritis patients. Sci Transl Med. 2015;7(290):290ra87.

13. Yeste A, et al. Tolerogenic nanoparticles inhibit T cell-mediated autoimmunity through SOCS2. Sci Signal. 2016;9(433):ra61.

14. Maldonado RA, et al. Polymeric synthetic nanoparticles for the induction of antigen-specific immunological tolerance. Proc Natl Acad Sci USA. 2015;112(2):E156-E165.

15. Tostanoski LH, et al. Reprogramming the Local Lymph Node Microenvironment Promotes Tolerance that Is Systemic and Antigen Specific. Cell Rep. 2016;16(11):2940-2952.

16. Capini C, et al. Antigen-specific suppression of inflammatory arthritis using liposomes. J Immunol. 2009;182(6):3556-3565.

17. van der Woude D, et al. Epitope spreading of the anti-citrullinated protein antibody response occurs before disease onset and is associated with the disease course of early arthritis. Ann Rheum Dis. 2010;69(8):1554-1561.

18. Olson SW, et al. Asymptomatic autoantibodies associate with future anti-glomerular basement membrane disease. $J$ Am Soc Nephrol. 2011;22(10):1946-1952. 
19. Legoux FP, et al. CD4+ T Cell Tolerance to Tissue-Restricted Self Antigens Is Mediated by Antigen-Specific Regulatory T Cells Rather Than Deletion. Immunity. 2015;43(5):896-908.

20. Malhotra D, et al. Tolerance is established in polyclonal CD4(+) T cells by distinct mechanisms, according to self-peptide expression patterns. Nat Immunol. 2016;17(2):187-195.

21. Vitali C, et al. Migratory, and not lymphoid-resident, dendritic cells maintain peripheral self-tolerance and prevent autoimmunity via induction of iTreg cells. Blood. 2012;120(6):1237-1245.

22. Idoyaga J, et al. Specialized role of migratory dendritic cells in peripheral tolerance induction. J Clin Invest. 2013;123(2):844-854.

23. Jakubzick C, Bogunovic M, Bonito AJ, Kuan EL, Merad M, Randolph GJ. Lymph-migrating, tissue-derived dendritic cells are minor constituents within steady-state lymph nodes. J Exp Med. 2008;205(12):2839-2850.

24. Sixt M, et al. The conduit system transports soluble antigens from the afferent lymph to resident dendritic cells in the $\mathrm{T}$ cell area of the lymph node. Immunity. 2005;22(1):19-29.

25. Kuan EL, et al. Collecting lymphatic vessel permeability facilitates adipose tissue inflammation and distribution of antigen to lymph node-homing adipose tissue dendritic cells. J Immunol. 2015;194(11):5200-5210.

26. Guo R, et al. Inhibition of lymphangiogenesis and lymphatic drainage via vascular endothelial growth factor receptor 3 blockade increases the severity of inflammation in a mouse model of chronic inflammatory arthritis. Arthritis Rheum. 2009;60(9):2666-2676

27. Campbell IK, et al. Differentiation of inflammatory dendritic cells is mediated by NF-kB1-dependent GM-CSF production in CD4 T cells. J Immunol. 2011;186(9):5468-5477.

28. Thomas SN, et al. Impaired humoral immunity and tolerance in K14-VEGFR-3-Ig mice that lack dermal lymphatic drainage. J Immunol. 2012;189(5):2181-2190.

29. Park K. The drug delivery field at the inflection point: Time to fight its way out of the egg. J Control Release. 2017;267:2-14.

30. Ghaffar KA, Giddam AK, Zaman M, Skwarczynski M, Toth I. Liposomes as nanovaccine delivery systems. Curr Top Med Chem. 2014;14(9):1194-1208

31. Maradana MR, et al. Immunomodulatory liposomes targeting liver macrophages arrest progression of nonalcoholic steatohepatitis. Metab Clin Exp. 2018;78:80-94.

32. Nelson KM, Dahlin JL, Bisson J, Graham J, Pauli GF, Walters MA. The Essential Medicinal Chemistry of Curcumin. J Med Chem. 2017;60(5):1620-1637.

33. Dong X, et al. Direct transcriptional regulation of RelB by 1alpha,25-dihydroxyvitamin D3 and its analogs: physiologic and therapeutic implications for dendritic cell function. J Biol Chem. 2003;278(49):49378-49385.

34. Dong $X$, et al. Regulation of relB in dendritic cells by means of modulated association of vitamin $\mathrm{D}$ receptor and histone deacetylase 3 with the promoter. Proc Natl Acad Sci USA. 2005;102(44):16007-16012.

35. Bally M, Mayer L, Hope M, Nayar R. Pharmacodynamics of liposomal drug carriers: methodological considerations. In: Gregoriadis eds. Liposome Technology, Florida, USA: CRC Press Inc. 1993:31.

36. Hayakawa M, Kobayashi M, Hoshino T. Direct contact between reticular fibers and migratory cells in the paracortex of mouse lymph nodes: a morphological and quantitative study. Arch Histol Cytol. 1988;51(3):233-240.

37. Junt T, et al. Subcapsular sinus macrophages in lymph nodes clear lymph-borne viruses and present them to antiviral B cells. Nature. 2007;450(7166):110-114.

38. Gerner MY, Casey KA, Kastenmuller W, Germain RN. Dendritic cell and antigen dispersal landscapes regulate T cell immunity. J Exp Med. 2017;214(10):3105-3122.

39. Martinez RJ, et al. Arthritogenic self-reactive CD4+ T cells acquire an FR4hiCD73hi anergic state in the presence of Foxp3+ regulatory T cells. J Immunol. 2012;188(1):170-181.

40. Mann EH, Chambers ES, Chen YH, Richards DF, Hawrylowicz CM. 1 $\alpha$,25-dihydroxyvitamin D3 acts via transforming growth factor- $\beta$ to up-regulate expression of immunosuppressive CD73 on human CD4+ Foxp3- T cells. Immunology. 2015;146(3):423-431

41. Carmona-Rivera C, et al. Synovial fibroblast-neutrophil interactions promote pathogenic adaptive immunity in rheumatoid arthritis. Sci Immunol. 2017;2(10):eaag3358.

42. van Lieshout AW, et al. Inhibition of TNF alpha during maturation of dendritic cells results in the development of semi-mature cells: a potential mechanism for the beneficial effects of TNF alpha blockade in rheumatoid arthritis. Ann Rheum Dis. 2005;64(3):408-414

43. Reynolds G, et al. Synovial CD4+ T-cell-derived GM-CSF supports the differentiation of an inflammatory dendritic cell population in rheumatoid arthritis. Ann Rheum Dis. 2016;75(5):899-907.

44. Garcia-Bates TM, et al. Contrasting Roles of the PD-1 Signaling Pathway in Dendritic Cell-Mediated Induction and Regulation of HIV-1-Specific Effector T Cell Functions. J Virol. 2019;93(5):e02035-18.

45. Buzás EI, Végvári A, Murad YM, Finnegan A, Mikecz K, Glant TT. T-cell recognition of differentially tolerated epitopes of cartilage proteoglycan aggrecan in arthritis. Cell Immunol. 2005;235(2):98-108.

46. Glant TT, et al. Proteoglycan-induced arthritis and recombinant human proteoglycan aggrecan G1 domain-induced arthritis in BALB/c mice resembling two subtypes of rheumatoid arthritis. Arthritis Rheum. 2011;63(5):1312-1321.

47. Korganow AS, et al. From systemic T cell self-reactivity to organ-specific autoimmune disease via immunoglobulins. Immunity. 1999;10(4):451-461.

48. Ooi JD, et al. The HLA-DRB1*15:01-restricted Goodpasture's T cell epitope induces GN. J Am Soc Nephrol. 2013;24(3):419-431.

49. Ooi JD, et al. Dominant protection from HLA-linked autoimmunity by antigen-specific regulatory T cells. Nature. 2017;545(7653):243-247.

50. Hu X, Kim H, Stahl E, Plenge R, Daly M, Raychaudhuri S. Integrating autoimmune risk loci with gene-expression data identifies specific pathogenic immune cell subsets. Am J Hum Genet. 2011;89(4):496-506.

51. Trynka G, et al. Chromatin marks identify critical cell types for fine mapping complex trait variants. Nat Genet. 2013;45(2):124-130

52. Nielen MM, et al. Increased levels of C-reactive protein in serum from blood donors before the onset of rheumatoid arthritis. Arthritis Rheum. 2004;50(8):2423-2427.

53. Nielen MM, et al. Specific autoantibodies precede the symptoms of rheumatoid arthritis: a study of serial measurements in blood donors. Arthritis Rheum. 2004;50(2):380-386. 
54. Scally SW, et al. A molecular basis for the association of the HLA-DRB1 locus, citrullination, and rheumatoid arthritis. $J$ Exp Med. 2013;210(12):2569-2582.

55. Hutton HL, Holdsworth SR, Kitching AR. ANCA-Associated Vasculitis: Pathogenesis, Models, and Preclinical Testing. Semin Nephrol. 2017;37(5):418-435.

56. O'Sullivan BJ, Thomas R. CD40 ligation conditions dendritic cell antigen-presenting function through sustained activation of NF-kappaB. J Immunol. 2002;168(11):5491-5498.

57. Nasreen M, Waldie TM, Dixon CM, Steptoe RJ. Steady-state antigen-expressing dendritic cells terminate CD4+ memory T-cell responses. Eur J Immunol. 2010;40(7):2016-2025.

58. Gottschalk RA, Corse E, Allison JP. TCR ligand density and affinity determine peripheral induction of Foxp3 in vivo. J Exp Med. 2010;207(8):1701-1711.

59. Latchman Y, et al. PD-L2 is a second ligand for PD-1 and inhibits T cell activation. Nat Immunol. 2001;2(3):261-268.

60. Grimm AJ, Kontos S, Diaceri G, Quaglia-Thermes X, Hubbell JA. Memory of tolerance and induction of regulatory T cells by erythrocyte-targeted antigens. Sci Rep. 2015;5:15907.

61. Fukaya T, et al. Crucial roles of B7-H1 and B7-DC expressed on mesenteric lymph node dendritic cells in the generation of anti gen-specific CD4+Foxp3+ regulatory T cells in the establishment of oral tolerance. Blood. 2010;116(13):2266-2276.

62. Shiokawa A, Kotaki R, Takano T, Nakajima-Adachi H, Hachimura S. Mesenteric lymph node CD11b CD103 ${ }^{+}$PD-L1 ${ }^{\text {High }}$ dendritic cells highly induce regulatory T cells. Immunology. 2017;152(1):52-64.

63. Bally AP, Austin JW, Boss JM. Genetic and Epigenetic Regulation of PD-1 Expression. J Immunol. 2016;196(6):2431-2437.

64. Shi J, Hou S, Fang Q, Liu X, Liu X, Qi H. PD-1 Controls Follicular T Helper Cell Positioning and Function. Immunity. 2018;49(2):264-274.e4.

65. Burton BR, et al. Sequential transcriptional changes dictate safe and effective antigen-specific immunotherapy. Nat Commun 2014;5:4741.

66. Barsony J, Renyi I, McKoy W, Kang HC, Haugland RP, Smith CL. Development of a biologically active fluorescent-labeled calcitriol and its use to study hormone binding to the vitamin D receptor. Anal Biochem. 1995;229(1):68-79.

67. Boyd BJ, Kaminskas LM, Karellas P, Krippner G, Lessene R, Porter CJ. Cationic poly-L-lysine dendrimers: pharmacokinetics, biodistribution, and evidence for metabolism and bioresorption after intravenous administration to rats. Mol Pharm. 2006;3(5):614-627.

68. Glant TT, et al. Proteoglycan-induced arthritis and recombinant human proteoglycan aggrecan G1 domain-induced arthritis in BALB/c mice resembling two subtypes of rheumatoid arthritis. Arthritis Rheum. 2011;63(5):1312-1321.

69. Glant TT, Mikecz K. Proteoglycan (aggrecan)-induced arthritis, A murine autoimmune model of rheumatoid arthritis. In: Perl A, ed. Autoimmunity: Methods and Protocols. Totowa, New Jersey, USA: Humana Press; 2004:313-38.

70. Ruutu M, et al. $\beta$-Glucan triggers spondylarthritis and Crohn's disease-like ileitis in SKG mice. Arthritis Rheum. 2012;64(7):2211-2222 